\title{
Predictive potential of Perzyna viscoplastic modelling for granular geomaterials
}

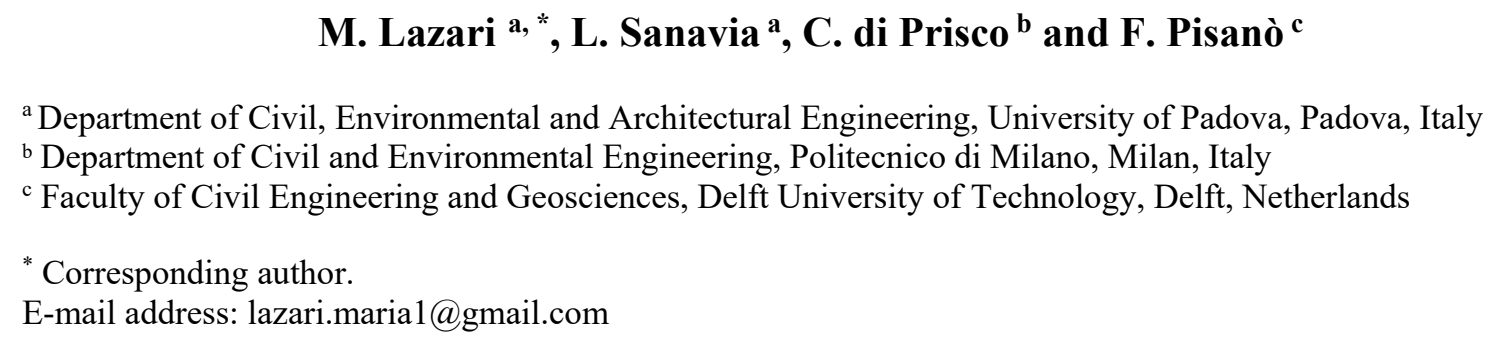

\section{ABSTRACT}

This paper reappraises Perzyna-type viscoplasticity for the constitutive modelling of granular geomaterials, with emphasis on the simulation of rate/time effects of different magnitude. An existing elasto-plastic model for sands is first recast into a Perzyna viscoplastic formulation, then calibrated/validated against laboratory test results on Hostun sand from the literature. Notable model features include (i) enhanced definition of the viscous nucleus function, and (ii) void ratio dependence of stiffness and viscous parameters, to model the pycnotropic behaviour of granular materials with a single set of parameters, uniquely identified against standard creep and triaxial test results. The comparison between experimental data and numerical simulations points out the predicative capability of the developed model and the complexity of defining a unique viscous nucleus function to capture sand behaviour under different loading/initial/boundary and drainage conditions. It is concluded that the unified viscoplastic simulation of both drained and undrained response is particularly challenging within Perzyna's framework and opens to future research in the area. The discussion presented is relevant, for instance, to the simulation of multi-phase strain localisation phenomena, such as those associated to slope stability problems in variably saturated soils.

Keywords: sand, creep, constitutive modelling, Perzyna viscoplasticity, strain localisation, regularisation

\section{INTRODUCTION}

There is wide experimental evidence of granular geomaterials responding to external perturbations rapidly but not instantaneously. The microstructural rearrangements that cause macroscopic deformations take place over time frames sometimes in the order of minutes - such as in the case of loose sands [1]. Laboratory investigations regarding strain rate effects, creep and relaxation in sand have been presented in [1]-[8]. In light of those experimental observations, the mathematical modelling of 
granular soil behaviour may be successfully tackled in the framework of delayed plasticity theories also referred to as viscoplasticity - or through a viscous evanescent relationship. Most viscoplastic models are formulated according to either of two different approaches: those allowing the stress state to lie outside the assumed elastic domain [9], [10] and those relying on rate-dependent hardening rules (consistency viscoplasticity, [11]-[14]). Alternatively, [15]-[16] proposed a viscous evanescent relationship within a general three-component model framework. Here, the former approach in the version proposed by Perzyna [9], [17] will be followed due to its proven capability to reproduce the ratedependence of both fine- and coarse-grained soils [18]-[21], including creep and relaxation phenomena. Importantly, Perzyna's viscoplasticity has also proven a suitable conceptual platform for the interpretation of several soil instabilities ([19], [22]-[25]).

Viscoplasticity has also gained further success over the years as a regularisation technique for strain localisation simulations. This notable property relates to the intrinsic characteristic length possessed by viscoplastic media as a consequence of their time-sensitiveness ([11], [22], [26]-[30]). As a consequence, the ill-posedness of inviscid elasto-plastic problems at the onset of bifurcation can be remedied [31], as well as the pathologic mesh-dependence of corresponding finite element simulation results. Enhanced regularisation performance has also been achieved via a non-local reformulation of standard viscoplastic constitutive equations (e.g. [32], [33]). Such approach is beneficial for materials whose viscosity-related characteristic length is physically inaccurate, or in fact too small to produce any regularisation.

This work addresses the less investigated problem of formulating/calibrating viscoplastic models that can quantitatively capture the rate-dependent behaviour of sands under diverse loading/initial/boundary conditions. For this purpose, the existing elasto-plastic sand model by Buscarnera and Nova [34] (based on the previous work of Jommi [35], Jommi and di Prisco [36] and Nova et al. [37]) is reformulated according to Perzyna's viscoplastic approach and validated against experimental data from the literature. Special attention is devoted to the importance of the viscous nucleus definition - main factor affecting the rate-sensitiveness - and to the intrinsic pycnotropy of sand behaviour (dependence on the void ratio). The latter aspect is addressed by introducing a straightforward void-ratio dependence of certain soil parameters (viscosity, stiffness and hardening coefficients), as already explored e.g. by [38].

The ultimate goal of the work is to re-open a discussion on the fundamental requisites of viscoplastic sand models, not solely in terms of their regularisation performance, but primarily of their physical soundness predictive capability. 
The proposed elasto-viscoplastic model is formulated hereafter under the assumption of isotropic hydro-mechanical behaviour, also including the effects of variation in suction and/or degree of saturation for generality. The model builds upon the isotropic hardening formulation for unsaturated soils proposed in [34], based on the previous work of [35]-[37]. Direct notation is adopted, with boldface and lightface italic symbols denoting tensors/vectors and scalars, respectively.

\section{$2.1 \quad$ Stress/strain variables and elastic law}

The small-strain multiaxial formulation of the model is based on the following definition of the generalised effective Cauchy stress tensor $\boldsymbol{\sigma}^{\prime}$ [39], applicable to three-phase porous materials with incompressible solid grains:

$$
\boldsymbol{\sigma}^{\prime}=\boldsymbol{\sigma}-S_{\mathrm{w}} p^{\mathrm{w}} \mathbf{1}-\left(1-S_{\mathrm{w}}\right) p^{\mathrm{g}} \mathbf{1}
$$

where $\sigma$ is the total Cauchy stress tensor, $p^{w}$ and $p^{g}$ the pressures of pore liquid water and gas, $S_{w}$ the water degree of saturation, and $\mathbf{1}$ the second-order identity tensor. The cases of dry and water saturated soil are recovered by setting $S_{w}=0$ and $S_{w}=1$, respectively.

79 The total strain rate is decomposed additively into elastic/reversible $\left(\dot{\boldsymbol{\varepsilon}}^{\mathrm{e}}\right)$ and viscoplastic/irreversible ( $\dot{\boldsymbol{\varepsilon}}^{\mathrm{vp}}$ ) components:

$$
\dot{\boldsymbol{\varepsilon}}=\dot{\boldsymbol{\varepsilon}}^{\mathrm{e}}+\dot{\boldsymbol{\varepsilon}}^{\mathrm{vp}}
$$

81 with the latter being by definition time-delayed. The elastic behaviour of the material emerges directly

82 from a strain energy function $\psi\left(\varepsilon^{\mathrm{e}}\right)$ :

$$
\boldsymbol{\sigma}^{\prime}=\frac{\partial \psi}{\partial \boldsymbol{\varepsilon}^{\mathrm{e}}}\left(\boldsymbol{\varepsilon}^{\mathrm{e}}\right)
$$

83 and can be than cast into the following rate form:

$$
\dot{\boldsymbol{\sigma}}^{\prime}=\mathbf{D}^{\mathrm{e}}\left[\dot{\boldsymbol{\varepsilon}}-\dot{\boldsymbol{\varepsilon}}^{\mathrm{vp}}\right]
$$

84 In Equation (4) $\mathbf{D}^{\mathrm{e}}$ is the fourth-order elastic stiffness tensor given by:

$$
\mathbf{D}^{\mathrm{e}}=\frac{\partial^{2} \psi\left(\boldsymbol{\varepsilon}^{\mathrm{e}}\right)}{\partial \boldsymbol{\varepsilon}^{\mathrm{e}} \otimes \partial \boldsymbol{\varepsilon}^{\mathrm{e}}}
$$

85 with $\psi\left(\boldsymbol{\varepsilon}^{\mathrm{e}}\right)$ being the same stored energy function already adopted by [37], [40], [41] - see Appendix 86 for more details. 


\subsection{Yield function and plastic potential}

Yield and plastic potential functions are defined as proposed in [42]:

$$
\left.\begin{array}{l}
f \\
g
\end{array}\right\}=A_{h}^{K-C^{\prime} / C_{h}} B_{h}^{-K_{2 h} / C_{h}} p^{\prime}-p_{s h}^{\prime}=0
$$

89

with

$$
\left.\begin{array}{l}
K_{1 h} \\
K_{2 h}
\end{array}\right\}=\frac{m_{h}\left(1-\alpha_{h}\right)}{2\left(1-m_{h}\right)}\left\{1 \pm \sqrt{1-\frac{4 \alpha_{h}\left(1-m_{h}\right)}{m_{h}\left(1-\alpha_{h}\right)^{2}}}\right\}
$$

90

$$
\begin{aligned}
& A_{h}=1+\frac{1}{K_{l h} M_{h}(\mathrm{~S})} \frac{q}{p^{\prime}} \\
& B_{h}=1+\frac{1}{K_{2 h} M_{h}(S)} \frac{q}{p^{\prime}} \\
& C_{h}=\left(1-m_{h}\right)\left(K_{l h}-K_{2 h}\right)
\end{aligned}
$$

91 where the subscript $h=f, g$ is used to indicate either the yield function or the plastic potential. In

92 Equations (6)-(8) $m_{h}$ and $\alpha_{h}$ are constitutive parameters (see Table 1). The interested reader is referred 93 to [34], [41], [42], where the physical meaning of relevant model parameters is described.

94 The current stress state is represented through the following three invariants:

$$
p^{\prime}=\frac{1}{3} \operatorname{tr}\left(\boldsymbol{\sigma}^{\prime}\right) \quad q=\sqrt{\frac{3}{2}}\|\mathbf{s}\| \quad S=\sin (3 \theta)=\sqrt{6} \frac{\operatorname{tr}\left(\mathbf{s}^{3}\right)}{\left[\operatorname{tr}\left(\mathbf{s}^{2}\right)\right]^{3 / 2}}
$$
in which $M_{c}$ and $M_{e}$ are the values of $M(S)$ associated with triaxial compression and extension, respectively. The internal variables $p_{s h}^{\prime}$ in Equation (6) govern the size of the yield locus and plastic where $p^{\prime}$ is the mean effective stress, $q$ the deviator stress (proportional to the norm of the deviator stress tensor s), and $S$ a trigonometric function of the Lode angle $\theta$ (with $\theta$ equal to $30^{\circ}$ in triaxial compression and $-30^{\circ}$ in triaxial extension). The variable $M_{h}(S)$ in Equation (8) is given after [43]:

$$
M_{h}(S)=\frac{2 c M_{c h}}{(1+c)-(1-c) S} \quad c=\frac{M_{e h}}{M_{c h}}
$$
potential in the effective stress space. In the following, $p_{s f}^{\prime}$ is simply denoted as $p_{s}^{\prime}$ and termed preconsolidation stress, while $p_{s g}^{\prime}$ is a dummy variable that does not affect the stress gradient of $g$. 


\subsection{Viscoplastic flow rule}

103 The rate of viscoplastic strains is obtained according to the well-known approach proposed by Perzyna

104 [9], [17]:

$$
\dot{\boldsymbol{\varepsilon}}^{\mathrm{vp}}=\gamma \Phi(f) \frac{\partial g}{\partial \boldsymbol{\sigma}^{\prime}}
$$

105 where $f$ and $g$ keep denoting yield and plastic potential functions (Equation (6)), and $\Phi$ is commonly

106 referred to as "viscous nucleus". In the same equation, the so-called "fluidity parameter" $\gamma$ governs the 107 rate-sensitiveness of the solid skeleton $(\gamma=1 / \eta$, with $\eta$ viscosity $)$ and specifically the rate at which 108 viscoplastic strains occur. Increasing $\gamma$ values reduce the rate-sensitiveness of the material: when $\gamma \rightarrow \infty$ 109 the mechanical response tends to its elasto-plastic (rate-insensitive) limit. At variance with rate110 independent plasticity, the magnitude of the viscoplastic strain rate results directly from the scalar 111 "distance" $\Phi$ between the current stress point and the yield locus (overstress), with no enforcement of 112 the usual consistency condition. The direction of the instantaneous viscoplastic flow is still governed by 113 the gradient of the plastic potential $g$.

114 The selection of the viscous nucleus function is a distinctive feature of elasto-viscoplastic Perzyna 115 models. It must be formulated and calibrated to pursue best agreement with experimental data from 116 standard creep tests, particularly by mobilising in experiments different levels of overstress. It should be 117 noted that the shape of the viscous nucleus function (and associated parameters) is model-specific, i.e. 118 affected by all other (elasto-plastic) features of the model. A more comprehensive discussion on this 119 matter can be found in [1]. Herein, the implications of two different definitions are discussed:

120 - power-law viscous nucleus, most common in the literature [11]:

$$
\Phi(f)=\left\langle\left(\frac{f}{\left|p^{\prime}\right|}\right)^{\alpha}\right\rangle=\left\langle\bar{f}^{\alpha}\right\rangle
$$

121 - exponential viscous nucleus, initially proposed in [1] for loose sands:

$$
\Phi(f)=\mathrm{e}^{\alpha \bar{f}}
$$

122 where $\alpha$ is in the above definitions an additional viscous parameter controlling the shape of the viscous

123 nucleus function (Table 1). The Macaulay brackets $<>$ are used in Equation (12) according their usual 124 meaning:

$$
\left\langle\bar{f}^{\alpha}\right\rangle= \begin{cases}\bar{f}^{\alpha} & \text { if } \bar{f}^{\alpha} \geq 0 \\ 0 & \text { if } \bar{f}^{\alpha}<0\end{cases}
$$


to make irreversible viscoplastic strains only occur when the stress state lies outside the elastic domain

126 (i.e. when $f>0$ ). Both expressions (12) - (13) fulfil the relevant theoretical requirements discussed by

127 [1], while the use of the dimensionless yield function $\bar{f}$ is appropriate for pressure-sensitive materials 128 [44], [45].

\subsection{Hardening rule}

130 Under general hydro-mechanical loading paths, the preconsolidation stress $p_{\mathrm{s}}^{\prime}$ evolves according to 131 the following hardening rule [34], [35]:

$$
\dot{p}_{\mathrm{s}}^{\prime}=\rho_{\mathrm{s}} p_{\mathrm{s}}^{\prime}\left(\dot{\varepsilon}_{v}^{v p}+\xi_{\mathrm{s}} \dot{\varepsilon}_{s}^{v p}\right)-r_{\mathrm{sw}} p_{\mathrm{s}}^{\prime} \dot{S}_{\mathrm{w}}
$$

132

133

\subsection{Influence of relative density}

139 Granular materials respond to mechanical perturbations depending on the current void ratio

140 (pycnotropy) and effective confining pressure (barotropy). This essential feature has been successfully

141 captured in the literature through the notion of "state parameter", which enables to reproduce the 142 behaviour of loose-to-dense materials with a single set of parameters [46]-[51].

143 Herein, the simpler approach proposed in [38] has been preferred to exploit the lack of the so-called 144 consistency condition. Accordingly, it is possible to incorporate pycnotropy into the viscoplastic 145 formulation by modulating certain constitutive parameters according to the current relative density (or 146 void ratio). This allows to describe the main consequences of dense-to-loose transitions (and vice versa), 147 such as softening and vanishing dilatancy at medium/large strains [32], [38]. As originally proposed in 148 [38], a linear dependence on the relative density is assumed here for the viscosity $\eta$, the representative 149 elastic shear modulus $\left(\mathrm{G}_{0}\right)$ and the hardening parameter $\mathrm{r}_{\mathrm{sw}}$ : 


$$
p_{\mathrm{i}}=p_{L i}+\left(p_{D i}-p_{L i}\right) \cdot D_{r}
$$

150 in which the value of the generic parameter $p_{i}$ depends on the current relative density $D_{r}$ and two 151 bounding values, $p_{L i}$ and $p_{D i}$, set for the loosest and densest reference conditions - here $D_{r}=20 \%$ and $152 D_{r}=100 \%$ respectively. Current $D_{r}$ value is updated at each integration step based on the evolving soil 153 volumetric strain [38]. However, such a linear dependence should not be taken for granted, and indeed 154 the following non-linear relationship has been found to perform better for the constitutive parameters $\xi_{s}$ 155 and $\rho_{s}$ (see Section 4.1 and [52]):

$$
p_{\mathrm{i}}=p_{L i}+\left(p_{D i}-p_{L i}\right) D_{r}^{5\left(e_{\max }-e_{\min }\right)}
$$

156

157

158

159

where in this case $p_{i}$ represents either $\xi_{s}$ or $\rho_{s}$, while $e_{\max } / e_{\min }$ are the maximum/minimum void ratios of the sand. In the spirit of the present viscoplastic approach, pycnotropy can be simply reproduced through density-dependent parameters. Nonetheless, specific $D_{r}$-dependences need to be identified by comparison to experimental results, and may assume the forms exemplified by Equations (18)-(19).

A synopsis of all constitutive parameters and their meaning is given in Table 1. The model as formulated above is suited for hydro-mechanical processes involving unsaturated conditions and viscous effects. Its performance in presence of strain localisation problems may be fully regularised by coupling viscoplasticity and extension to non-locality ([53]-[55]). This can be easily achieved through a nonlocal reformulation of the viscoplastic flow-rule Equation (11), as successfully attempted in a few previous works of the authors ([32], [33], [44], [38], [56]-[58]).

The following sections address the calibration and validation of the proposed constitutive model against the response of clean Hostun sand. Although conceived for generally unsaturated sands, the model will be solely tested for either saturated or dry conditions, due to the dearth of test data regarding the rate-sensitiveness of unsaturated sands. The goal is to investigate to what extent a single set of elastic, plastic and viscous parameters can be identified to capture sand response over a wide range of relative density, initial/drainage conditions, loading rate and stress paths.

The model described above has been implemented in the finite element code for multiphase porous media Comes-Geo, developed at the University of Padova ([33], [59]-[66]). All the numerical results have been obtained via explicit forward Euler stress-point integration [67], [68] after preliminary verification of the numerical implementation (see [52]) against the simulation results in Buscarnera and Nova (2009) ${ }^{1}$.

\footnotetext{
${ }^{1}$ It is always possible to compare the performance of a viscoplastic model to the response of its elasto-plastic (rate-insensitive) counterpart by simply setting a sufficiently high fluidity parameter $\gamma$ in Equation (11).
} 
178 All constitutive parameters have been calibrated based on literature triaxial and creep tests on Hostun 179 sand, allowing a separate identification of elasto-plastic and viscous parameters, respectively. Table 2

180 summarises the main features (drainage, initial confinement and void ratio) of the reference tests from 181 [50], [1], [8], [69]-[70] - the same test labels as in the original publications have been kept in the 182 following.

\subsection{Elasto-plastic parameters}

184 The parameters governing the elasto-plastic behaviour have been calibrated by assuming a very high

185 loading rate, i.e. by artificially forcing the response of the viscoplastic model towards its rate-insensitive

186 limit. For this purpose the triaxial test results labelled in Table 2 as hos 027 d4, batr02 and alert 9 have

187 been best-matched as exemplified in Figure 1. The final set of calibrated elasto-plastic parameters is

188 reported in Table 3 and Table 4, with the latter providing the loose-to-dense range of $D_{r}$-dependent 189 parameters.

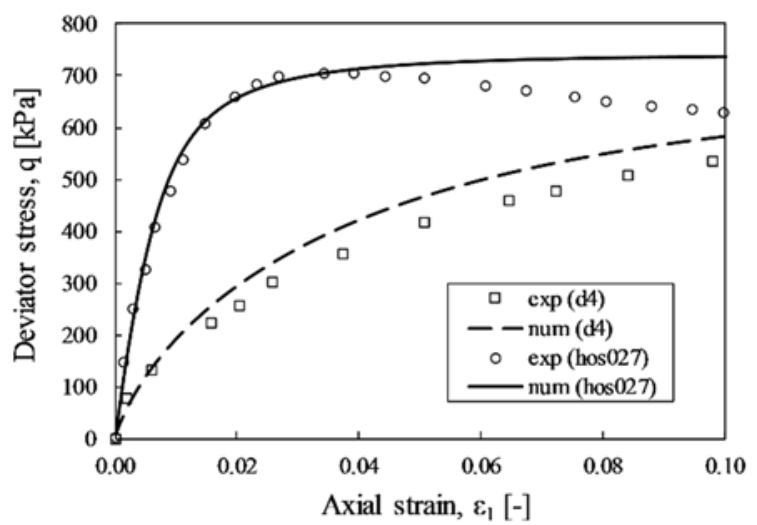

(a)

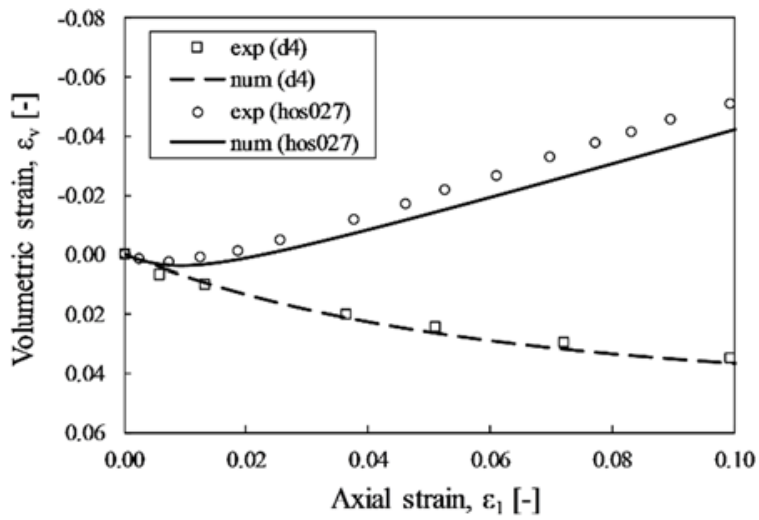

(c)

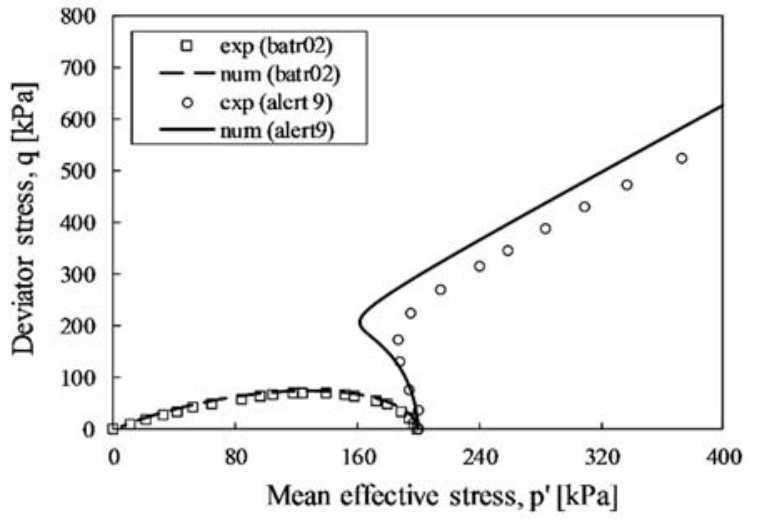

(b)

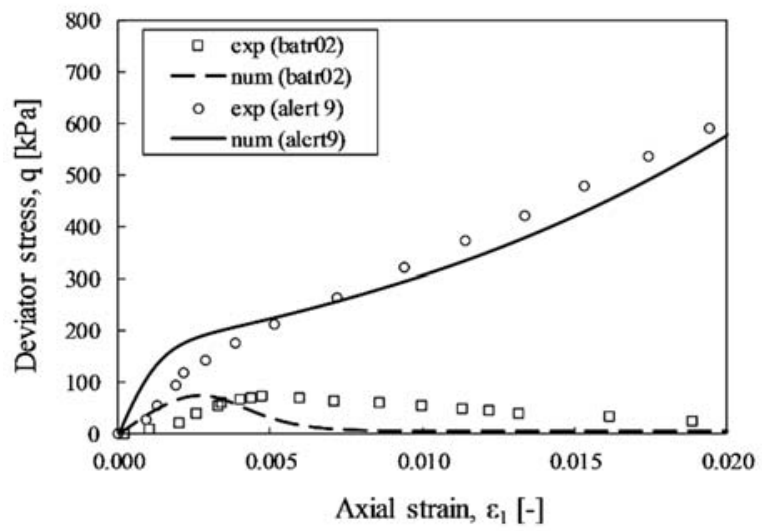

(d) 
Figure 1: Calibration of elasto-plastic parameters against (a), (c) drained and (b), (d) undrained triaxial test results for loose $(d 4$, batr02) and dense (hos027, alert 9$)$ Hostun sand.

191

192

193

194

195

\subsection{Viscous parameters}

With the same set of elasto-plastic parameters (Tables 3-4), the viscous parameters of Hostun loose and dense sand have been separately identified through the drained creep test results from [1] and [8]. di Prisco and Imposimato [1] performed tests on loose Hostun sand $\left(D_{r}=20 \%\right)$ by holding the radial effective stress constant while increasing the axial component up to attain the target stress obliquity; subsequent axial stress increments have been then applied with a five minutes time lag to explore the creep response. Two sets of viscous parameters have been calibrated for the different viscous nucleus definitions in Equations (12)-(13), namely linear ( $\alpha=1$ in the power-law expression) and exponential. With reference to the last creep step in the original publication (approximately 15 minutes duration), Figure 2 shows the axial strain vs time performance of the model (solid lines) in comparison to the experimental data from [1] (circular markers). It is readily apparent that satisfactory agreement can be achieved in this case regardless of the adopted viscous nucleus, as long as suitable (and nucleus-specific) viscous parameters are set (see Table 5).

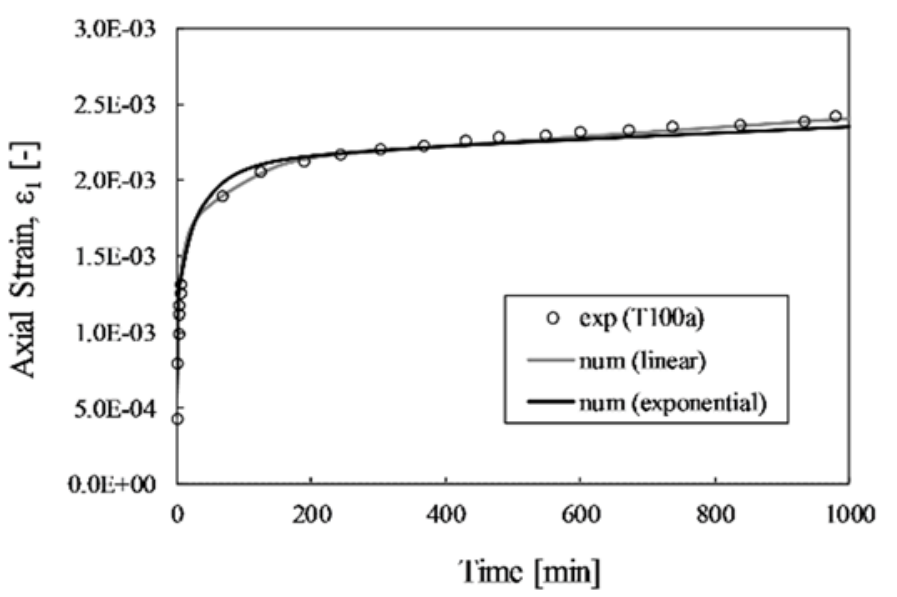

Figure 2: Simulation of loose Hostun sand creep by using linear and exponential viscous nuclei $\left(e_{0}=0.950, p^{\prime}=100 \mathrm{kPa}\right)$ : axial strain vs time.

The viscous parameters for Hostun dense sand $\left(D_{r}=71 \%{ }^{2}\right)$ have been then derived based on the experimental results from [8] and reported in Table 6. It should be noted that the same value of $\alpha$ (i.e. same shape of $\Phi$ ) has been used for both loose and dense Hostun sand (see Table 5 and Table 6) to reduce the number of free parameters. Such assumption is reasonably confirmed by data/simulations presented herein for Hostun sand, although future confirmation for different materials is needed. The

${ }^{2}$ Given the low viscosity of dense sands, the viscosity identified for $D_{r}=71 \%$ has been used as $p_{D}$ in Eq. (18). 
214 laboratory experiments were performed by initially consolidating the sample under an effective mean 215 pressure of $80 \mathrm{kPa}$, then followed by drained triaxial compression; the triaxial compression load path 216 included three additional stages of creep and cyclic loading, as illustrated in Figure 3.

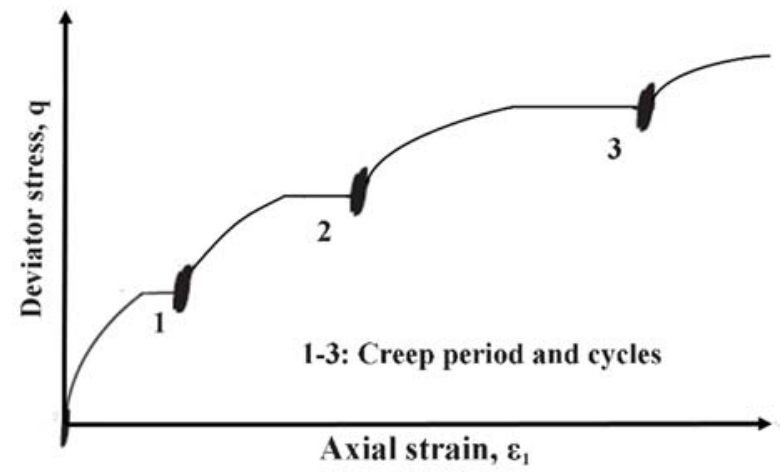

Figure 3: Creep test on dense Hostun sand (after [8]).

218 Experimental and numerical stress-strain curves are presented in Figure 4a for all four creep stages - no 219 intermediate cyclic loading simulated. For clearer visualisation in Figure 4b, the numerical axial strains 220 developed during creep after each triaxial compression are compared to the experimental results only 221 for the case of exponential viscous nucleus.

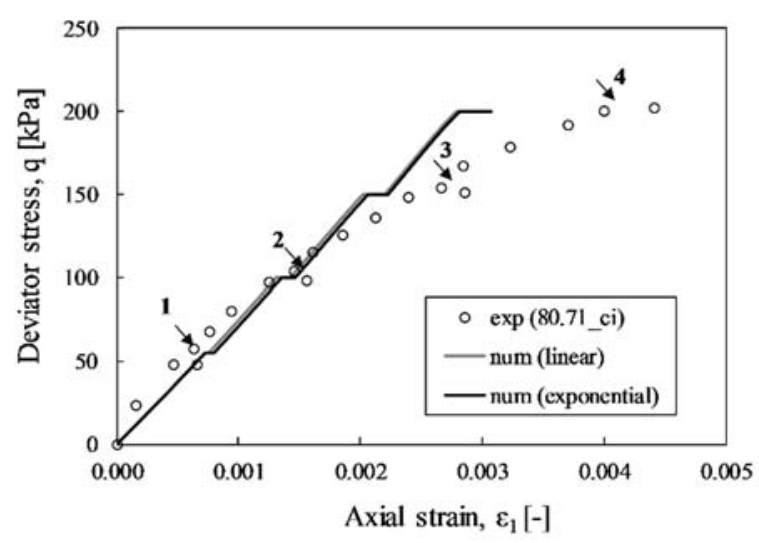

(a)

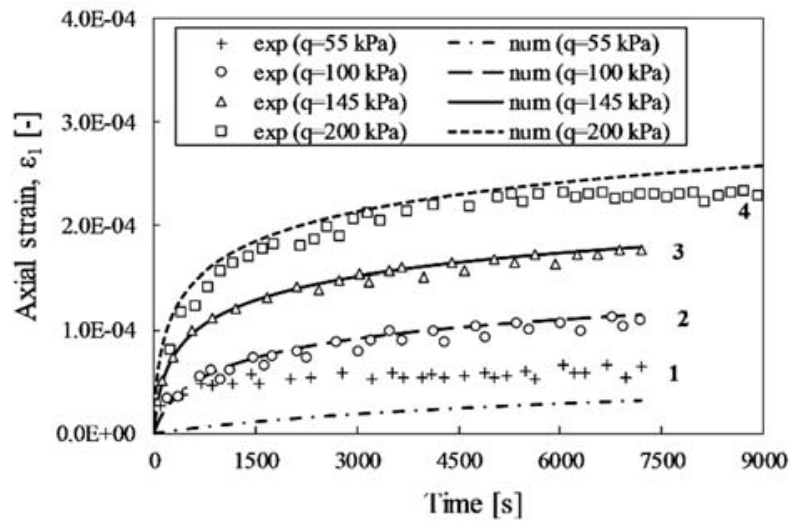

(b)

Figure 4: Simulation of creep behaviour for dense Hostun sand $\left(e_{0}=0.710, p^{\prime}=80 \mathrm{kPa}\right)$ : (a) global stress-strain response and (b) time evolution of axial strain.

The viscoplastic model reproduces with sufficient accuracy the response to all creep stages, though with some visible deviations from the global stress-strain behaviour (possibly affected by neglecting intermediate cyclic loading in the numerical simulations). Comparing the creep responses of loose and

225 dense Hostun sands points out more significant time effects for the former - in expected agreement with 226 the experimental literature. 
228 After the above parameter calibration, the full elasto-viscoplastic model is validated against the data set overviewed in Table 2. Such a validation is carried out for Hostun sand at two levels: (i) against triaxial 230 test results already used for the calibration of elasto-plastic parameters (Section 3.1); (ii) against different 231 experimental results - not previously considered - to produce valuable blind predictions. It should be

232 noted that the validation level (i) is still necessary to check whether the parameters derived from creep 233 tests produce appropriate time-sensitiveness when combined with different loading rate and test 234 conditions. The suitability of assuming $D_{r}$-dependent constitutive parameters is also highlighted in this 235 section.

236 All numerical simulations have been performed at imposed displacement rates of $1 \mathrm{~mm} / \mathrm{min}$ and 2 $\mathrm{mm} / \mathrm{min}$ for drained and undrained triaxial tests, respectively [72] - unless differently specified.

\subsection{Drained triaxial compression tests (TXD)}

The model is first validated against the experimental results of TXD tests at varying relative density and effective confinement. The predicted responses arising from the above viscous nucleus definitions are also critically compared, with all relevant material parameters listed in Table 3 to Table 6 . A linear

$242 D_{r}$-dependence (Equation (18)) is in some instances applied to all variable parameters, so as to point out 243 the better performance the non-linear relationship (19) conclusively applied to the parameters $\xi_{s}$ and $\rho_{s}$.

244 Further insight into the accuracy of the model is provided by comparison to the elasto-plastic predictions 245 obtained through the kinematic-hardening constitutive model of Gajo and Wood [50].

\subsubsection{Loose Hostun sand}

247 The outcomes of the two different viscous nuclei (Equations (12)-(13)) are compared in Figure 5 for 248 the TXD response of a loose sample ( $d 4$ in Table 2 ). The results clearly witness the superior performance 249 of the non-linear/exponential viscous nucleus, which confirms the quite complex dependence of sand 250 viscosity on the overstress level (i.e. on the value of $f$ ). It should be noted that the mismatching TXD 251 predictions in Figure 5 come after the same level of accuracy achieved by both viscous nuclei in slow creep tests (Figure 2). This conclusion is also confirmed by the cases of medium dense and dense Hostun sand discussed in the following. 


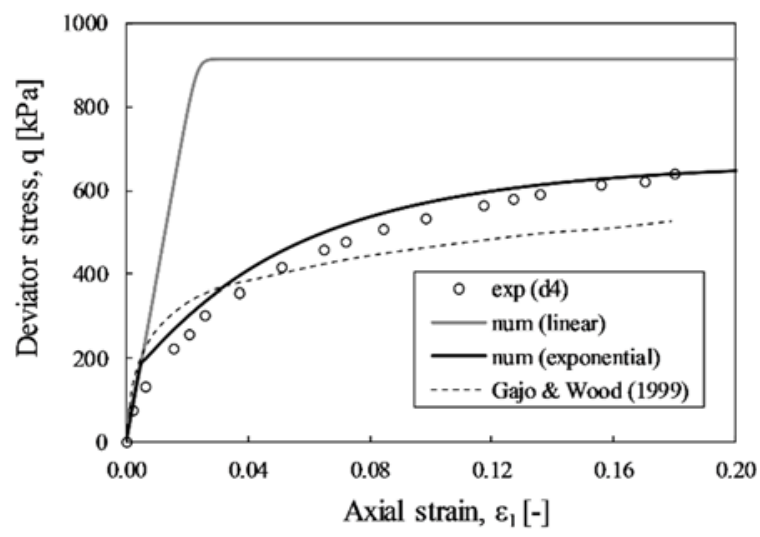

(a)

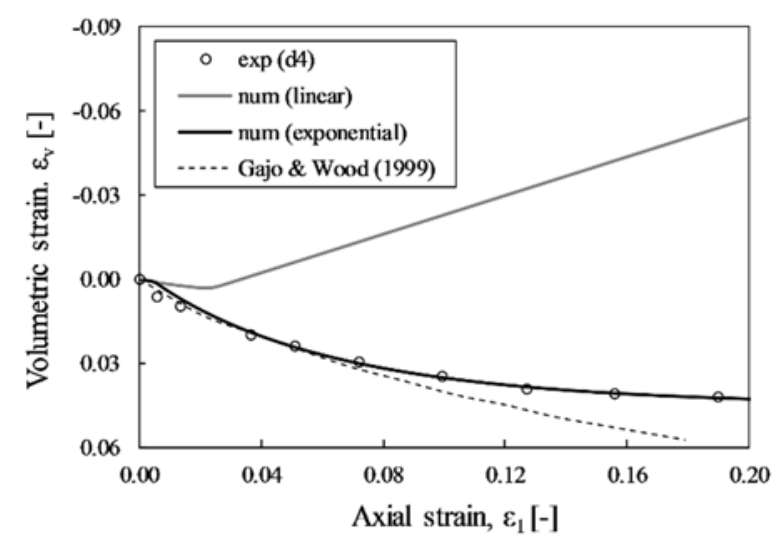

(b)

Figure 5: TXD test on loose Hostun sand $\left(e_{0}=0.945, p_{0}^{\prime}=300 \mathrm{kPa}\right)$ : (a) deviatoric stress-strain response and (b) volumetric behaviour.

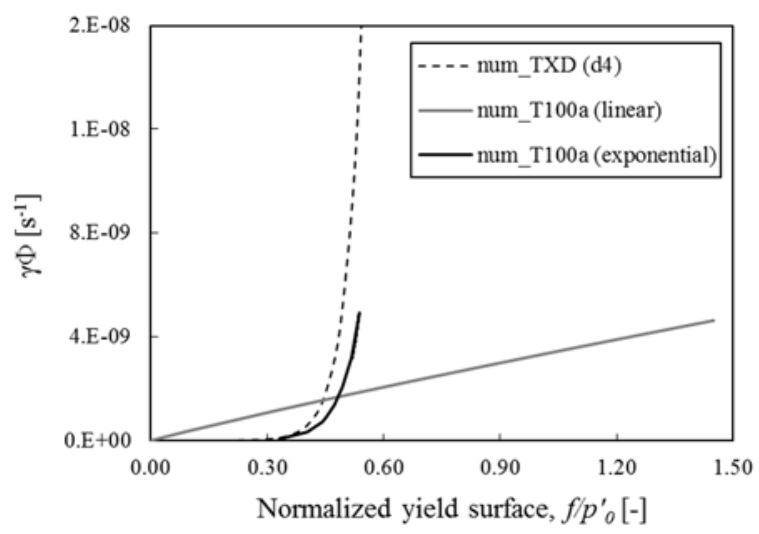

Figure 6: Evolution of the $\gamma \Phi$ product for creep (T100a) and TXD (d4) tests on loose Hostun sand.

255 The applicability of the exponential viscous nucleus is underpinned by Figure 6, where the evolution of

256 the $\gamma \Phi$ product (fluidity parameter times viscous nucleus) is plotted against the yield function values for the above TXD (d4) and creep (T100a) tests. Apparently, the linear viscous nucleus leads to very high values of the yield surface, at variance with the exponential formulation. This stems from the interaction

259 between the functions assumed in this study for the viscous nucleus and the yield locus [42], with the

260 latter being in turn a (very) non-linear function of the (over)stress state. The effect of such interaction

261 stands out under high(er) overstress levels, therefore more clearly under traxial loading than during 262 creep.

\subsubsection{Dense Hostun sand}

264 The comparison between experimental and numerical dense sand behaviour is illustrated in Figure 7

265 (hos027 in Table 2). As in the loose sand case, the results from the linear viscous nucleus are quite 266 unsatisfactory: the peak stress is significantly underestimated (Figure 7a), while the predicted volumetric 
strain trend is less dilative than in reality (Figure 7b). Conversely, the use of exponential nucleus allows 268 to capture correctly both the peak stress and the dilatancy, although the strain softening behaviour can 269 only be reproduced via the non- linear $D r$-dependence of $\xi_{s}$ and $\rho_{s}$ (Equation (19)).

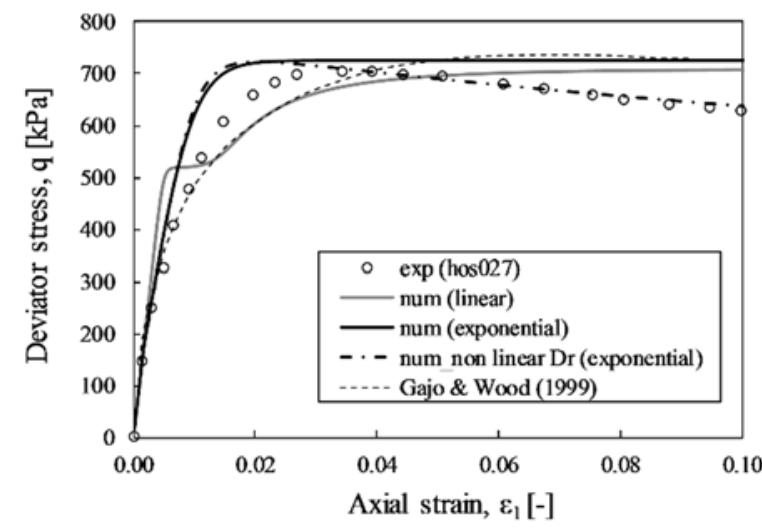

(a)

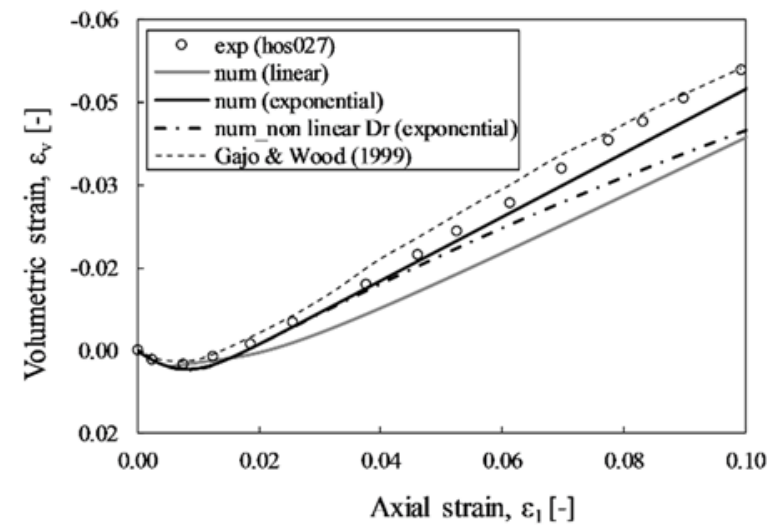

(b)

Figure 7: TXD test on dense Hostun sand $\left(e_{0}=0.578, p^{\prime}=200 \mathrm{kPa}\right)$ : (a) deviatoric stress-strain response and (b) volumetric behaviour.

\subsubsection{Medium dense Hostun sand}

271 The TXD tests on medium-dense Hostun sand are well simulated by the model enhanced with non272 linear $D_{r}$-dependence - see Figure 8 . The peak deviator stress is perfectly matched in Figure 8a, as well 273 as the overall volumetric response in Figure $8 \mathrm{~b}$.

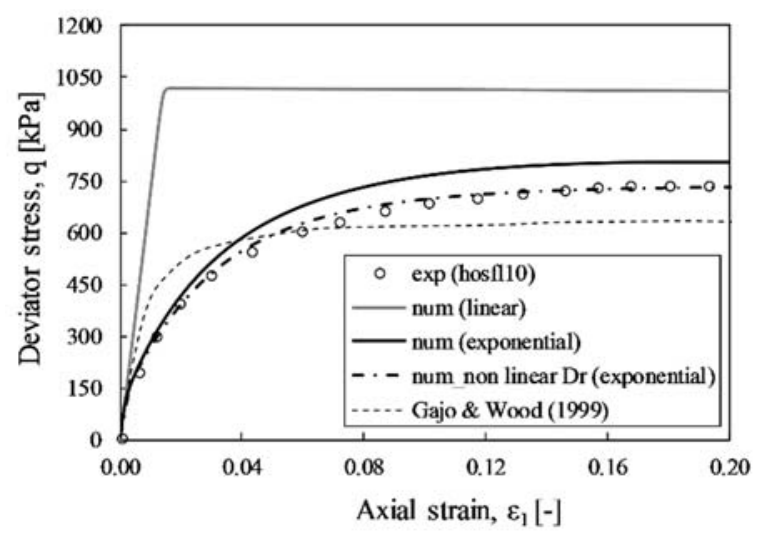

(a)

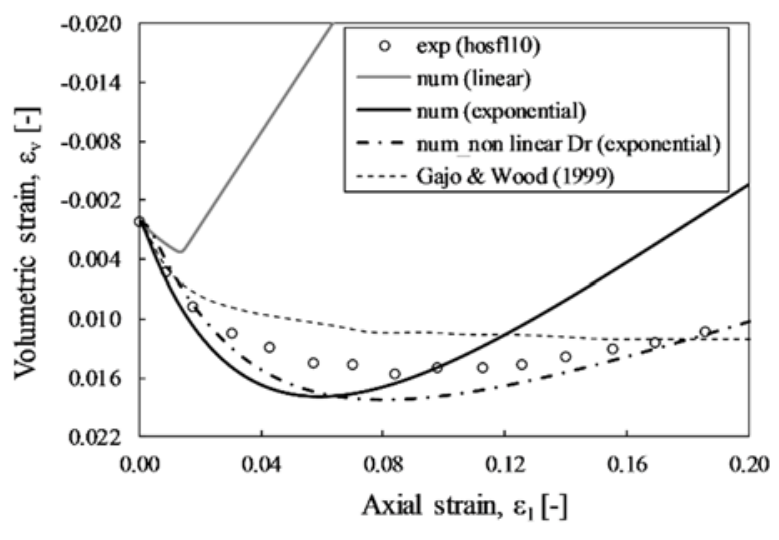

(b)

Figure 8: TXD test on medium-dense Hostun sand $\left(e_{0}=0.8, p^{\prime}{ }_{0}=300 \mathrm{kPa}\right)$ : (a) deviatoric stress-strain response and (b) volumetric behaviour.

\subsubsection{Further TXD model predictions}

Additional TXD predictions are reported hereafter to further validate the viscoplastic model in its final version with exponential viscous nucleus and non-linear $D_{r}$-dependence of the hardening parameters in

277 Equation (19). Experimental vs numerical comparisons are given in Figure 9 for TXD tests on loose 

- experimental data from [69].

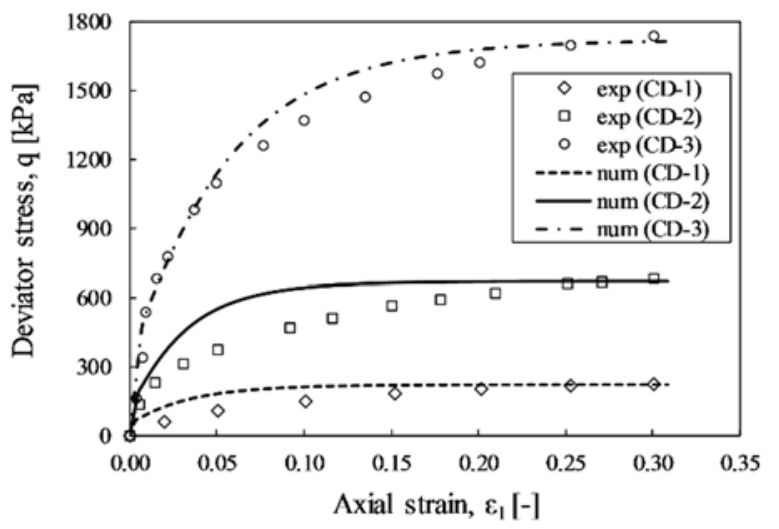

(a)

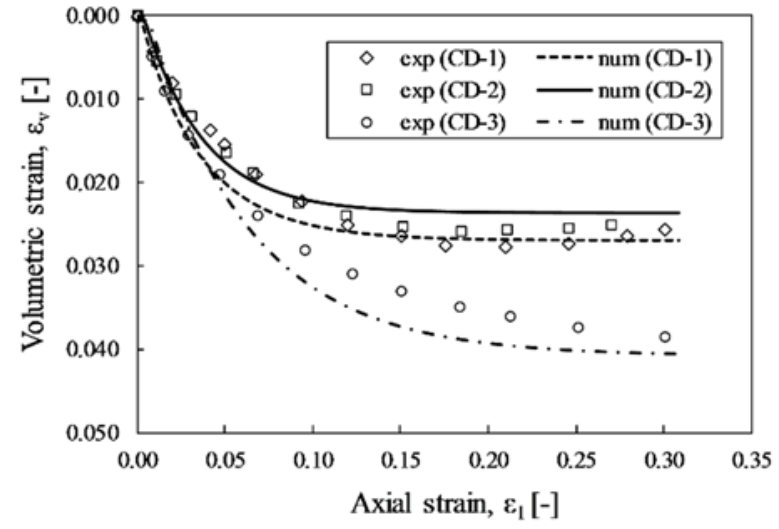

(b)

Figure 9: TXD tests on loose Hostun sand at varying effective confinement $\left(e_{0}=0.954-1.052\right.$, $p^{\prime}{ }_{0}=100,300,750 \mathrm{kPa}$ ): (a) deviatoric stress-stress response and (b) volumetric behaviour.

280 The stress-strain curves and volumetric trends in Figure 9 confirm that model can capture the intrinsic 281 pressure-dependence of sand behaviour at a given relative density ( $D r \approx 10 \%)$, with rate-effects spontaneously accommodated by the combination of suitable viscous parameters and realistic TXD loading rate. Relatedly, Figure 10 presents the model predictions obtained for dense and loose Hostun samples initially consolidated under the same isotropic pressure of $300 \mathrm{kPa}$. For the dense sample, both strain softening behaviour (Figure 10a) and dilation (Figure 10b) are satisfactorily simulated with respect to the experimental results from [50] - test hos011 in Table 2. Similar conclusions may be extended to the loose sample case - test hosfl11 (Figure 10c-d).

Figure 11, shows the case of two medium dense Hostun specimens initially consolidated at either low or medium/high effective pressures, $50 \mathrm{kPa}$ (test hosfl14) and $600 \mathrm{kPa}$ (test $h f l w 10$ ). As expected, the performance of the model is slightly worse - though not dramatically - for intermediate void ratios, for which more accurate modelling of barotropy/pycnotropy is likely needed.

292 The last TXD simulations in Figure 12 allow to further inspect the rate-sensitiveness of the monotonic 293 triaxial response. For this purpose, the experimental results on air-dried loose Hostun sand from [8] have 294 been considered, including isotropic consolidation up to $400 \mathrm{kPa}$ followed by axial straining at two 295 different rates - one 10 times larger than the other. Even though Perzyna-type models are necessarily 296 sensitive to the loading rate until the inviscid limit, such sensitivity must be quantitatively compared to 297 experimental evidence. In agreement with previous/related sources (see e.g. [3], [15]), the experimental results in Figure 12 confirm negligible rate-dependence of Hostun sand at the considered constant strain rates - note the almost coincident stress-strain curves. Elasto-viscoplastic simulations with unaltered 
constitutive parameters display in this case underpredicted sand stiffness, but confirm the observed low rate-sensitiveness of the material. The merit for the latter outcome comes mostly from the adopted

302 viscous nucleus formulation, suitable to capture (drained) rate-sensitiveness over a wide overstress 303 range.

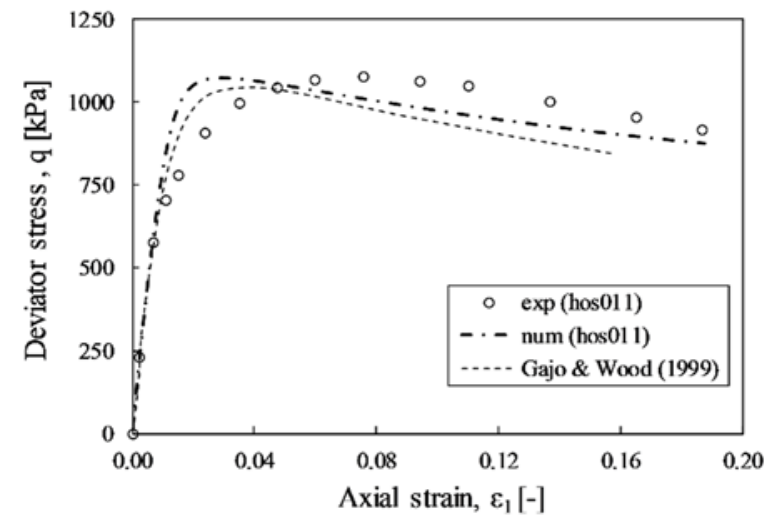

(a)

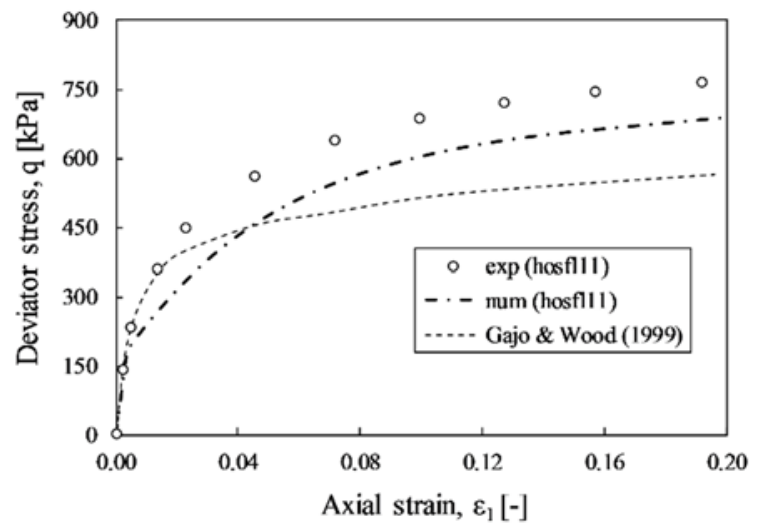

(c)

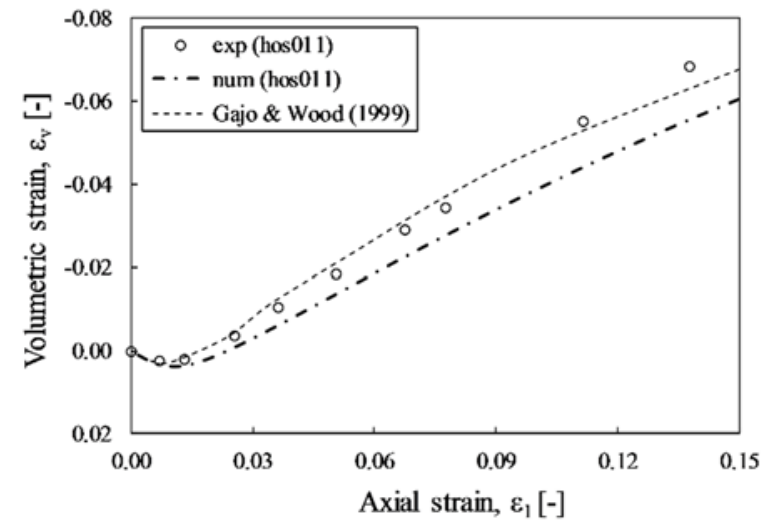

(b)

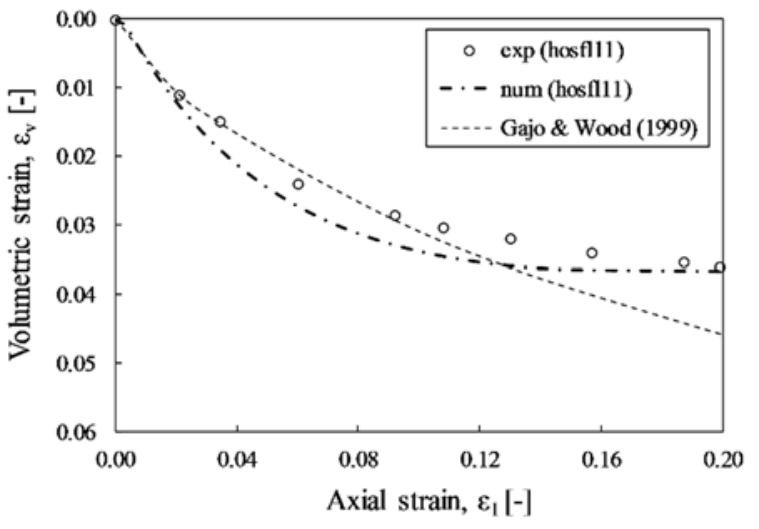

(d)

Figure 10: TXD tests on dense $\left(e_{0}=0.574\right)$ and loose $\left(e_{0}=0.897\right)$ Hostun sand at the same effective confinement ( $p_{0}^{\prime}=300 \mathrm{kPa}$ ): (a)-(c) deviatoric stress-stress response and (b)-(d) volumetric behaviour.

Overall, the results in this section show good ability of the model to reproduce TXD tests at varying 306 initial void ratio, effective confinement and loading rate, with viscous parameters independently identified from creep experiments. This achievement is not dramatically affected by the unavoidable heterogeneity of materials, facilities and operators in the reference experimental studies. 


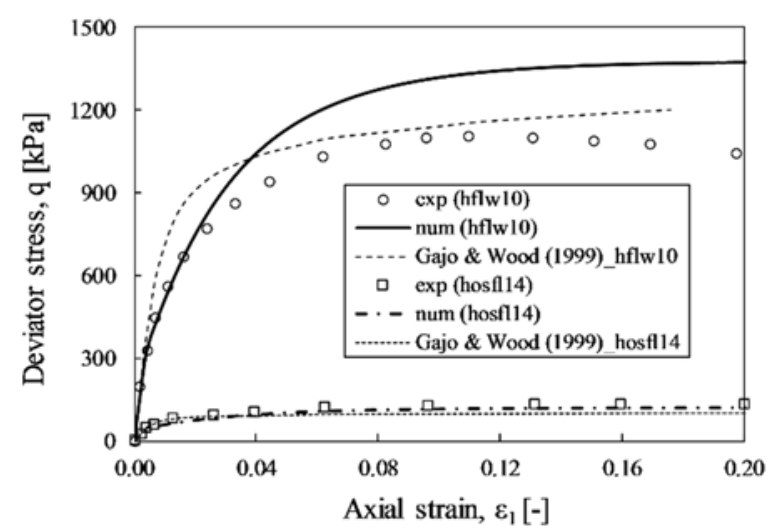

(a)

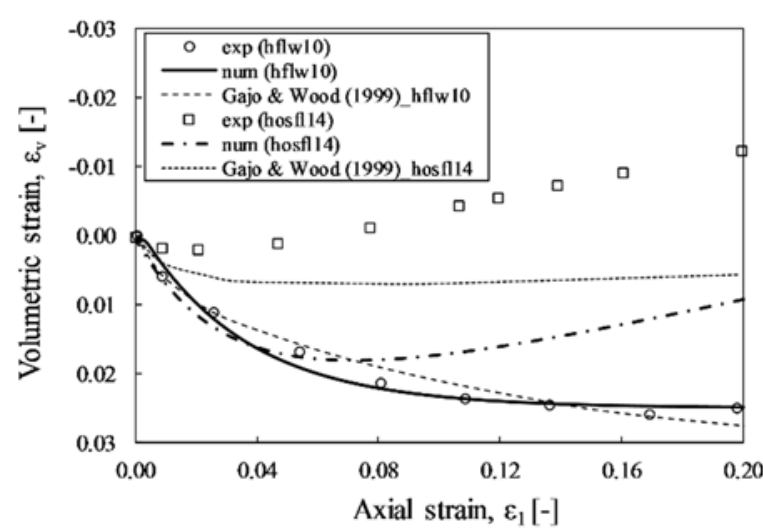

(b)

Figure 11: TXD tests on medium dense $\left(e_{0}=0.822-0.838\right)$ Hostun sand and different effective confinement $\left(p_{0}^{\prime}=50,600 \mathrm{kPa}\right)$ : (a) deviatoric stress-stress response and (b) volumetric behaviour.

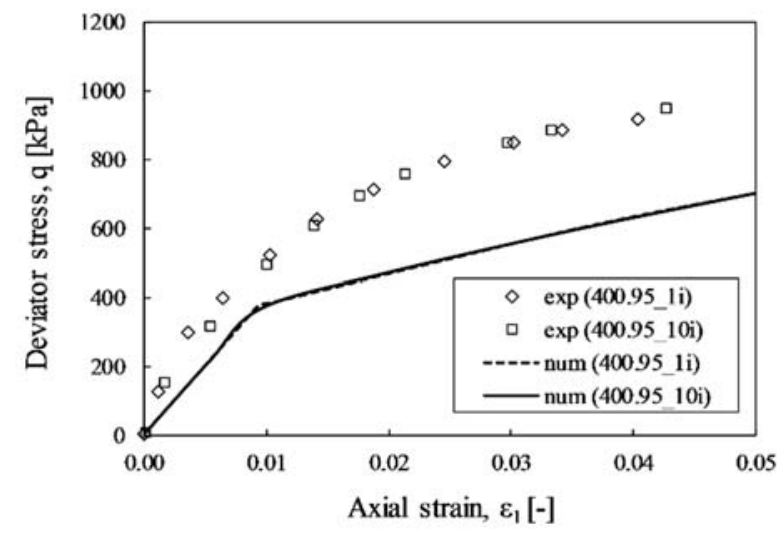

Figure 12: TXD on air-dried loose Hostun sand $\left(e_{0}=0.95\right)$ performed, respectively, at a strain rate of $\dot{\varepsilon}_{0}$ $=0.06 \% / \mathrm{min}$, test $400.951 \mathrm{i}$ and $\dot{\varepsilon}_{0}=0.6 \% / \mathrm{min}$, test $400.9510 \mathrm{i}\left(\right.$ data from $\left.[8]^{3}\right)$.

\subsection{Undrained triaxial compression tests (TXU)}

311 In this subsection the undrained triaxial performance of the proposed model is explored with respect

312 to the TXU tests in Table 2. It is noted that transiting to undrained conditions jeopardises the suitability

313 of the viscous parameters in Tables 5-6, which leads to envisage $\Phi(f)$ function probably more complex

314 than the assumed exponential form [56]. The different stress paths characterising TXD and TXU tests

315 mobilise different ranges of the $\Phi$-f relationship, whose non-linearity should be captured for accurate

316 simulations over a wide spectrum of loading conditions. Relatedly, simplistic viscous nucleus

${ }^{3}$ Intermediate relaxation branches in the original data have been removed from the plot, as overlooked in the numerical simulations for the sake of simplicity. 
formulation conceived, for instance, for numerical regularisation purposes, may yield misleading results 318 when applied to very diverse loading/drainage conditions.

319 The parameters of the exponential viscous nucleus have been thus recalibrated based on the TXU 320 results in Figures 13-14, for loose and dense Hostun sand respectively - tests batr02 and alert9 from 321 [50]. The same figures indicate that the new viscous parameters in Table 7 along with the above elasto322 plastic parameters (Tables 3-4) result in reasonable simulations of undrained stress paths and deviatoric 323 stress-stress responses for both loose and dense samples. It is also worth observing that the $D_{r^{-}}$ 324 dependence of constitutive parameters is here only relevant to setting proper initial conditions, as the 325 void ratio does not vary during TXU loading.

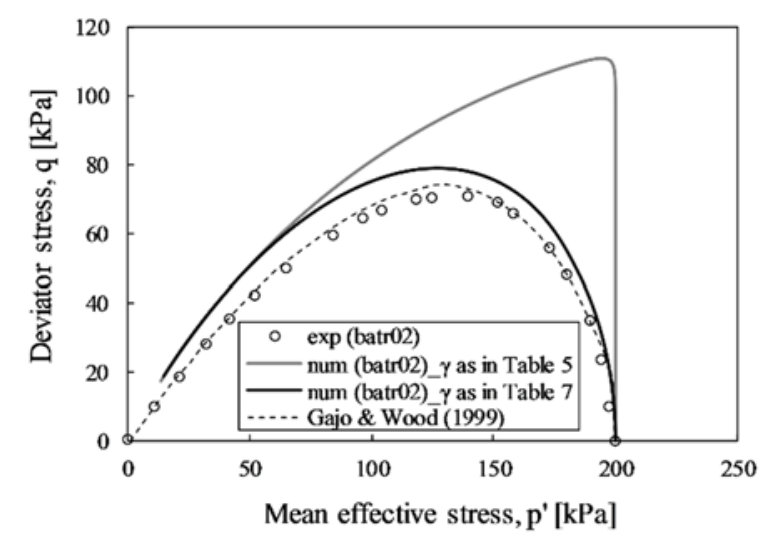

(a)

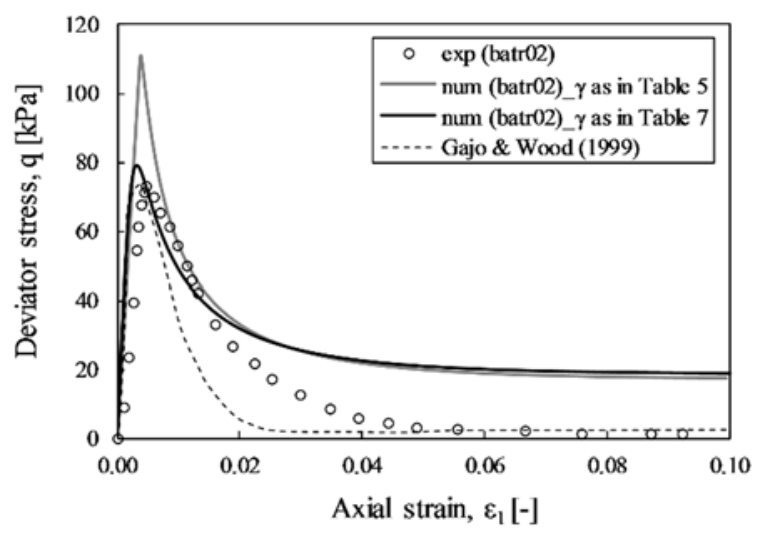

(b)

Figure 13: Re-calibration of the viscous parameters against TXU test on loose Hostun sand $\left(e_{0}=0.940, p_{0}^{\prime}=200 \mathrm{kPa}\right)$ : (a) stress path and (b) deviatoric stress-stain response.

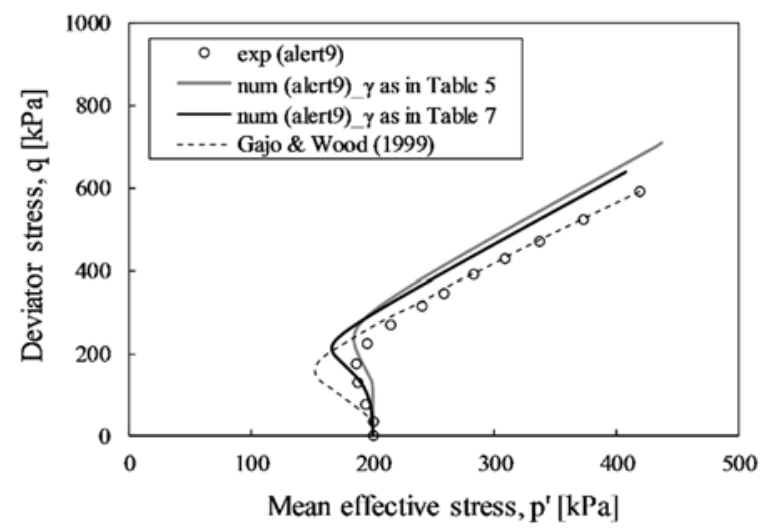

(a)

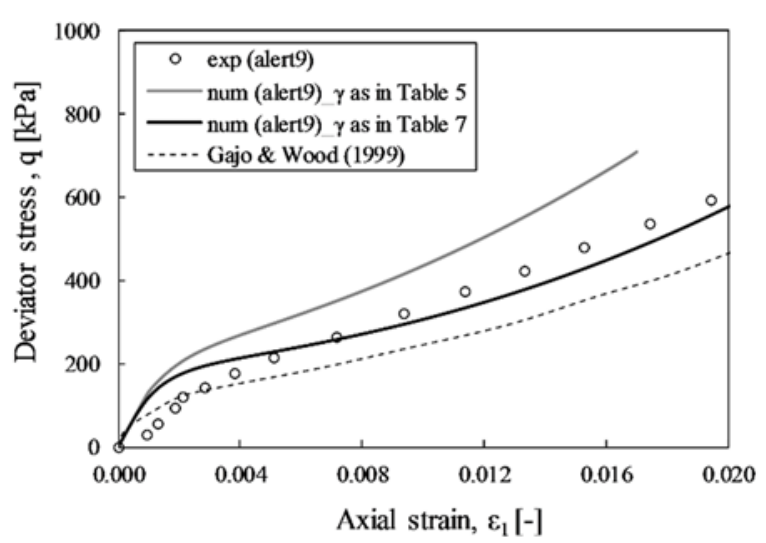

(b)

Figure 14: Re-calibration of the viscous parameters against TXU test on dense Hostun sand $\left(e_{0}=0.666, p^{\prime}=200 \mathrm{kPa}\right)$ : (a) stress path and (b) deviatoric stress-stain response. 
328 After the identification of "undrained" viscous parameters, TXU tests on loose and medium-dense 329 Hostun specimens have been considered for re-validation. The results in Figure 15 concern the tests 330 from [69] on loose sand at initial confinement equal to $750 \mathrm{kPa}(I C U-1), 300 \mathrm{kPa}(I C U-2)$ and $100 \mathrm{kPa}$ 331 (ICU-3). Encouraging numerical predictions have been found again in all relevant respects, and 332 particularly in terms of undrained stress path and pore pressure build-up. Similar satisfactory results can 333 be seen in Figure 16 for the medium dense sand tested by [50] - test batr06.

334

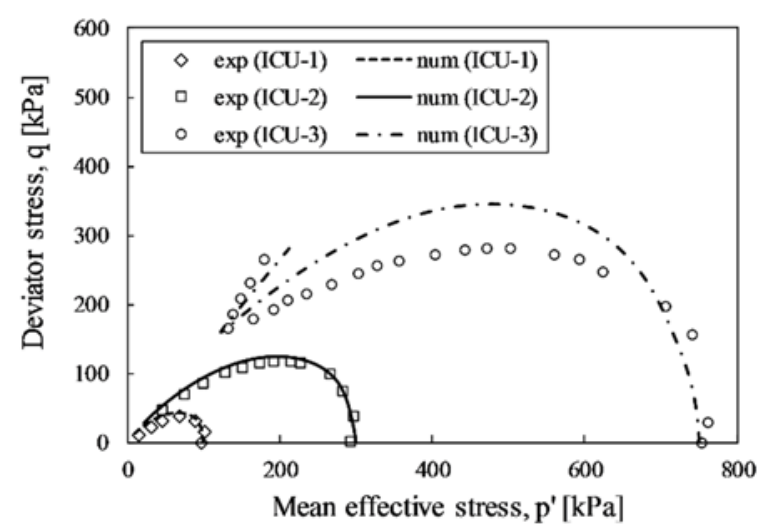

(a)

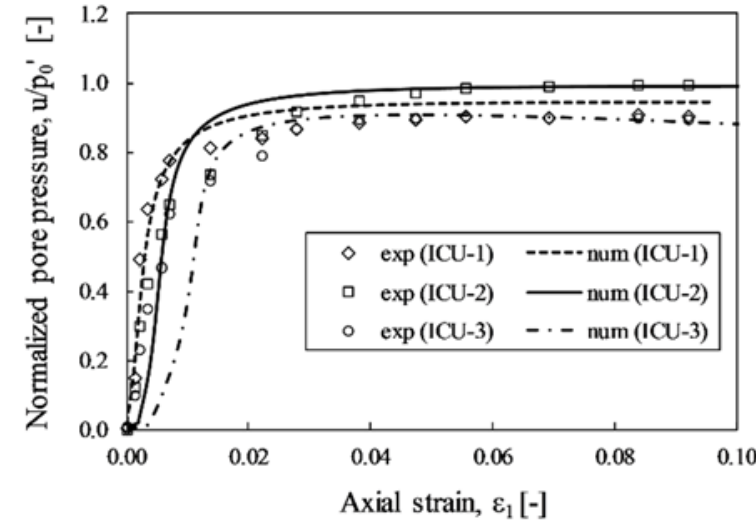

(b)

Figure 15: TXU tests on loose Hostun sand at varying effective confinement $\left(e_{0}=1.060-1.083\right.$, $p_{0}^{\prime}=100,300,750 \mathrm{kPa}$ ): (a) stress path and (b) normalized pore pressure versus axial strain.

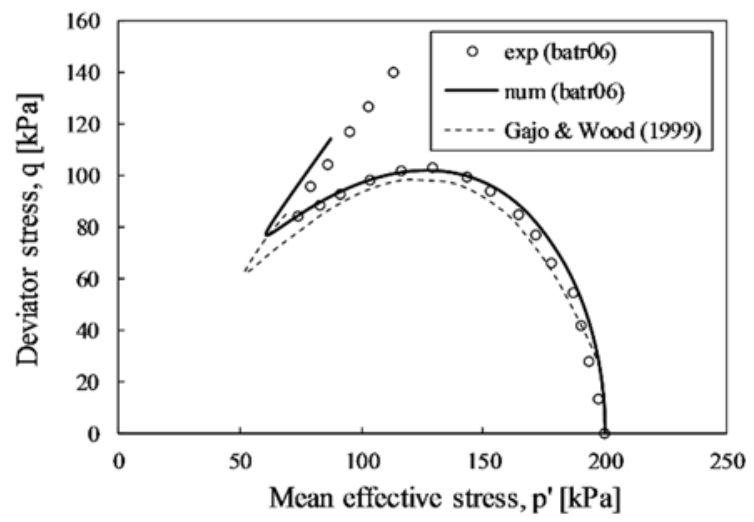

(a)

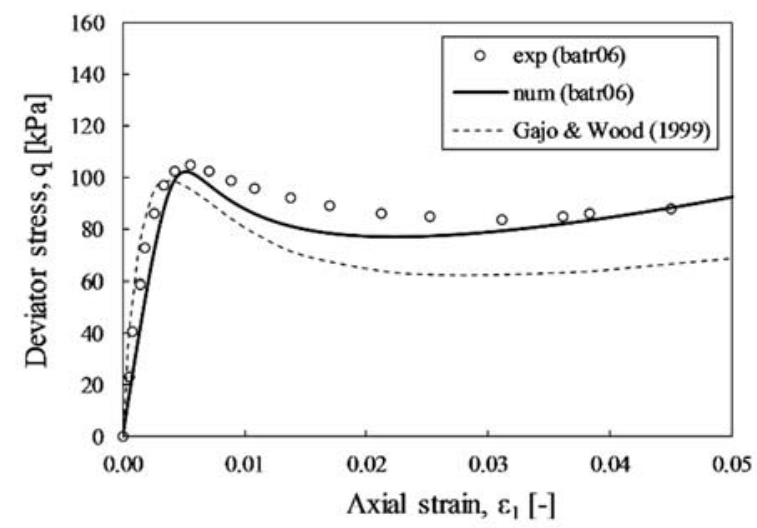

(b)

Figure 16: TXU on medium-dense $\left(e_{0}=0.830, p^{\prime}=200 \mathrm{kPa}\right)$ Hostun sand: (a) stress path and (b) deviatoric stress-stain response. 


\subsection{Undrained plane-strain/biaxial compression test}

338 Further validation has been successfully sought against the biaxial undrained test results documented

339 in [70] - test $S H F N D 05, p_{0}^{\prime}=800 \mathrm{kPa}$, axial displacement rate equal to $1.2 \mathrm{~mm} / \mathrm{min}$. Similarly to drained

340 conditions, the model endowed with its "undrained" viscous nucleus is capable to blindly predict other

341 experimental data not used for calibration. Specifically, Figure 17 illustrates the excellent agreement

342 achieved in terms of stress path, stress-strain response and pore pressure build up.

\section{$3434.4 \quad$ Undrained creep tests}

344 The results of (rare) undrained creep tests on Hostun loose sand are used as a final benchmark - data

345 from [71]. The original experimental tests were conducted with undrained creep following a preliminary

346 TXD stage up to target stress obliquity. Such a loading programme (test 20DP13) has been simulated

347 with the same parameters mentioned in Sections 4.2-4.3. During the TXD phase small load increments

348 were applied: between two subsequent load increments a time period of 5 minutes elapsed; when the

349 desired stress level was reached $\left(\mathrm{q}=61 \mathrm{kPa}, \mathrm{p}^{\prime}=120 \mathrm{kPa}\right)$ a further load increment of $2 \mathrm{kPa}$ was applied.

350 The predictions in Figure 18 obtained for the creep stage show reasonable agreement in terms of axial

351 strain and pore pressure. The premature onset of creep instability (inflection point in the pore pressure

352 curve) is most likely due to the specific yield function shape and the (simplistic) assumption of isotropic

353 hardening ([25], [75]), rather than to viscous modelling. 


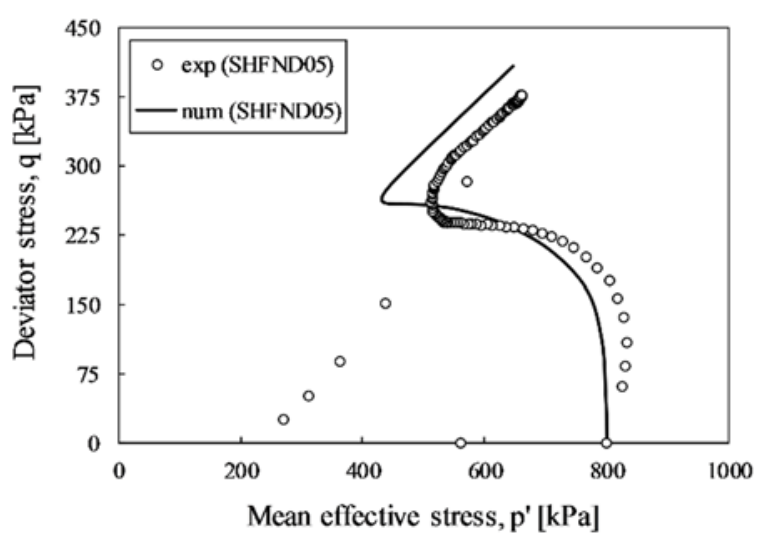

(a)

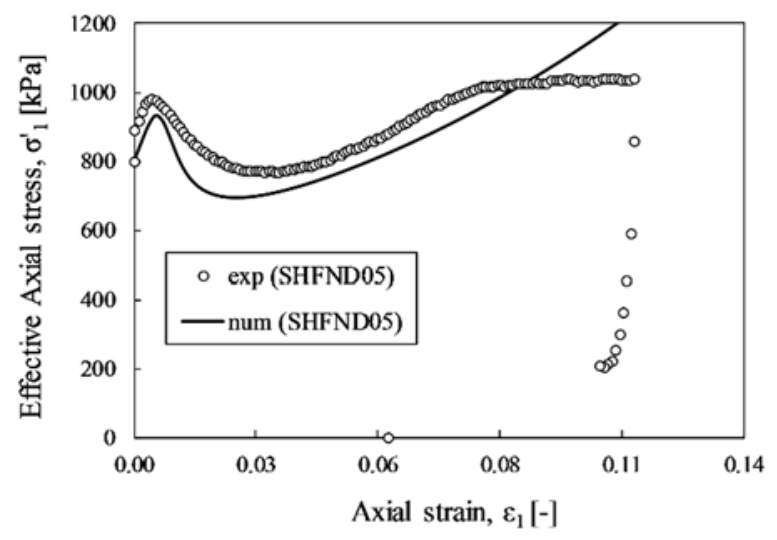

(b)

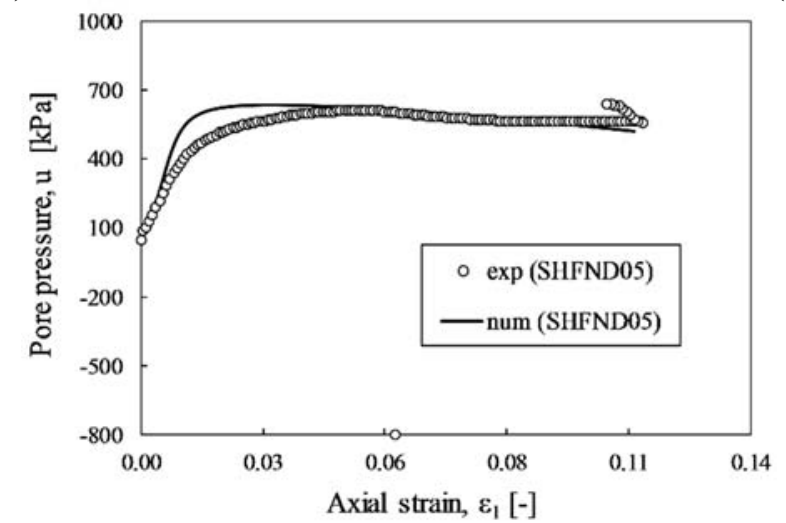

(c)

Figure 17: Biaxial test on loose Hostun sand $\left(e_{0}=0.945, p^{\prime}=800 \mathrm{kPa}\right)$ : (a) stress path, (b) deviatoric 354 stress-stain response, (c) pore pressure build-up.

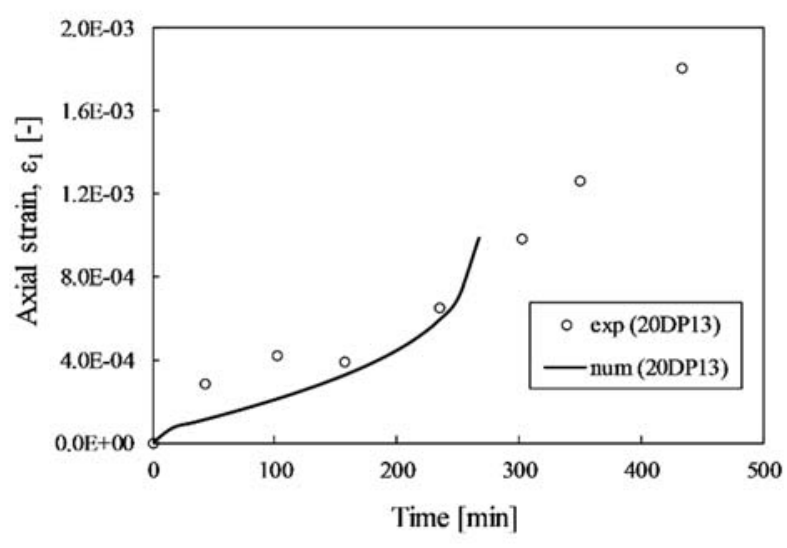

(a)

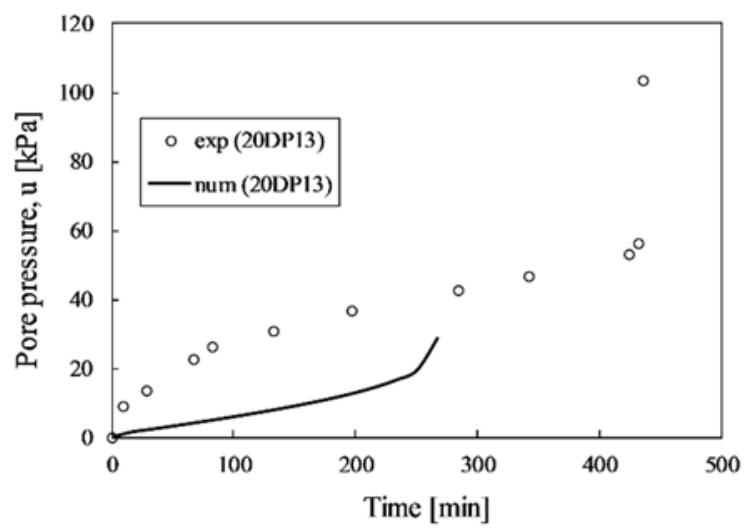

(b)

Figure 18: Undrained creep test on loose Hostun sand $\left(e_{0}=0.900, p^{\prime}=100 \mathrm{kPa}\right)$ : time evolution of (a) axial strain and (b) pore pressure. 
357 An existing elasto-plastic model for sandy soils was reformulated as a Perzyna viscoplastic relationship to capture the rate-sensitive, pycnotropic and barotropic behaviour of sands under different loading/initial/boundary and drainage conditions. In particular, the suitability of two alternative viscous nucleus definitions, namely linear and exponential, was verified with respect to both creep and triaxial test data on Hostun sand from the literature. Importantly, the parameters governing the time-dependence of the material were separately calibrated against creep tests and then found suitable to reproduce the different loading paths/rates induced during standard triaxial tests. While the need for quite complex viscous nucleus functions was confirmed, it was also shown how challenging still is to unify the simulation of both drained and undrained responses under a single analytical formulation with a unique set of material parameters. Unlike most literature on the subject, this work highlights that simplistic assumptions about rate-sensitiveness may abruptly reduce the predictive potential of elasto-viscoplastic models.

369 From a modelling perspective, it should be noted that quantitative conclusions on the predictive range are very specific of both the viscous nucleus and yield functions adopted. The non-linearity needed of the viscous nucleus $\Phi$ for good match with real data relates necessarily to how non-linear the $f$ function is. When inherited from existing elasto-plastic formulations for granular soils, capped yield loci and plastic potentials are most often very non-linear, as necessary to capture the response under diverse loading programmes (including e.g. radial stress paths). This fact not only makes extension to viscoplasticity less straightforward, but also poses conceptual questions about the effects of convexity losses experienced by these functions in the overstress regime (i.e. outside the $\mathrm{f}=0 / \mathrm{g}=0$ loci). Expected consequences might concern the predicted stability of the constitutive response [23], a subject so far never explored from this standpoint. When documented, convexity-related issues might be remedied by resorting to recent convexification techniques ([73], [74]).

380 The discussion offered in this work also aimed to discourage simplistic use of viscoplasticity as a mere numerical expedient against mesh-dependence in strain-localisation problems. Conversely, the viscoplastic framework was reappraised as a physically sound approach to sand modelling, easy to extend to non-locality whenever also characteristic length effects are relevant.

\section{ACKNOWLEDGMENTS}

385 The authors wish to thank the 7th Framework Programme of the European Union (ITN MuMoLaDe 386 project 289911) and the University of Padova for financially supporting this work. The valuable input 387 of Giuseppe Buscarnera and Constance Mihalache (Northwestern University) and Jacques Desrues 388 (Université de Grenoble-Alpes) is also gratefully acknowledged. 
Stored energy function and hyperelastic behaviour

392 The strain energy function $\psi\left(\varepsilon^{\mathrm{e}}\right)$ in Equation (5) is given by the following two-invariant expression:

$$
\psi\left(\varepsilon^{\mathrm{e}}\right)=\bar{\psi}\left(\varepsilon_{\mathrm{v}}^{\mathrm{e}}, \varepsilon_{\mathrm{s}}^{\mathrm{e}}\right)=\tilde{\psi}\left(\varepsilon_{\mathrm{v}}^{\mathrm{e}}\right)+\frac{3}{2}\left[G_{0}+\frac{\mathrm{a}}{\hat{k}} \tilde{\psi}\left(\varepsilon_{\mathrm{v}}^{\mathrm{e}}\right)\right]\left(\varepsilon_{\mathrm{s}}^{\mathrm{e}}\right)^{2}
$$

393 where:

$$
\tilde{\Psi}\left(\varepsilon_{\mathrm{v}}^{\mathrm{e}}\right)=\left\{\begin{array}{cl}
\hat{k} p_{r} \exp \left(\frac{\varepsilon_{\mathrm{v}}^{\mathrm{e}}}{\hat{k}}-1\right), \quad \varepsilon_{\mathrm{v}}^{\mathrm{e}} \geq \hat{k}\left(\text { or } p^{\prime} \geq p_{r}\right) \\
p_{r} \varepsilon_{\mathrm{v}}^{\mathrm{e}}+\frac{p_{r}\left(\varepsilon_{\mathrm{v}}^{\mathrm{e}}-\hat{k}\right)^{2}}{2 \hat{k}}, \quad \varepsilon_{\mathrm{v}}^{\mathrm{e}}<\hat{k}\left(\text { or } p^{\prime}<p_{r}\right)
\end{array}\right.
$$

394 This model produces pressure-dependent bulk and shear elastic moduli. $G_{0}, \hat{k}$, a are constitutive 395 parameters, $p_{r}$ is a reference mean effective stress, while $\varepsilon_{v}^{e}, \varepsilon_{s}^{e}$ are the elastic volumetric strain and the 396 second invariant of the elastic strain deviator, respectively. When $p^{\prime}<p_{r}$ the hyperelastic law predicts a 397 linear elastic behaviour, whereas a fully non-linear pressure dependent behaviour is obtained for $p^{\prime} \geq p_{r}$. 398 By taking the first and the second derivative of Equation (20) with respect to $\boldsymbol{\varepsilon}^{\mathrm{e}}$, the following 399 expressions for the stress and the elastic stiffness tensor are obtained:

$$
\boldsymbol{\sigma}^{\prime}=\frac{\partial \psi\left(\boldsymbol{\varepsilon}^{\mathrm{e}}\right)}{\partial \boldsymbol{\varepsilon}^{\mathrm{e}}}=\left[1+\frac{3 \mathrm{a}}{2 \hat{k}}\left(\varepsilon_{s}^{e}\right)^{2}\right] \theta_{\varepsilon} \mathbf{1}+2\left(G_{0}+\frac{\mathrm{a}}{\hat{k}} \tilde{\psi}\right) \mathbf{e}^{\mathrm{e}}
$$

400 and:

$$
\mathbf{D}^{\mathrm{e}}=\left[1+\frac{3 \mathrm{a}}{2 \hat{k}}\left(\varepsilon_{s}^{e}\right)^{2}\right] K_{\varepsilon} \mathbf{1} \otimes \mathbf{1}+2\left(G_{0}+\frac{\mathrm{a}}{\hat{k}} \tilde{\psi}\right)\left[\mathbf{I}-\frac{1}{3} \mathbf{1} \otimes \mathbf{1}\right]+2\left(\frac{\mathrm{a}}{\hat{k}}\right) \theta_{\varepsilon}\left(\mathbf{1} \otimes \mathbf{e}^{\mathrm{e}}+\mathbf{e}^{\mathrm{e}} \otimes \mathbf{1}\right)
$$

401 where, $\mathbf{e}^{\mathbf{e}}=\boldsymbol{\varepsilon}^{\mathbf{e}}-\frac{1}{3} \operatorname{tr}\left(\mathbf{\varepsilon}^{\mathbf{e}}\right) \mathbf{1}$ is the deviatoric elastic strain and:

$$
\theta_{\varepsilon}=\frac{\partial \tilde{\psi}\left(\varepsilon_{\mathrm{v}}^{\mathrm{e}}\right)}{\partial \varepsilon^{\mathrm{e}}}= \begin{cases}p_{r} \exp \left(\frac{\varepsilon_{\mathrm{v}}^{\mathrm{e}}}{\hat{k}}-1\right), & \varepsilon_{\mathrm{v}}^{\mathrm{e}} \geq \hat{k}\left(\text { or } p^{\prime} \geq p_{r}\right) \\ p_{r} \frac{\varepsilon_{\mathrm{v}}^{\mathrm{e}}}{\hat{k}}, & \varepsilon_{\mathrm{v}}^{\mathrm{e}}<\hat{k}\left(\text { or } p^{\prime}<p_{r}\right)\end{cases}
$$




$$
K_{\varepsilon}=\frac{\partial \theta_{\varepsilon}}{\partial \varepsilon^{\mathrm{e}}}= \begin{cases}\frac{p_{r}}{\hat{k}} \exp \left(\frac{\varepsilon_{\mathrm{v}}^{\mathrm{e}}}{\hat{k}}-1\right), & \varepsilon_{\mathrm{v}}^{\mathrm{e}} \geq \hat{k}\left(\text { or } p^{\prime} \geq p_{r}\right) \\ \frac{p_{r}}{\hat{k}}, & \varepsilon_{\mathrm{v}}^{\mathrm{e}}<\hat{k}\left(\text { or } p^{\prime}<p_{r}\right)\end{cases}
$$

403

404

405

406

407

408

409

410

411

412

413

414

415

416

417

418

419

420

421

422

423

424

425

426

427

428

429

430

431

432

433

434

\section{REFERENCES}

[1] di Prisco C, Imposimato S. Time dependent mechanical behaviour of loose sands. Mechanics of Cohesive-Frictional Materials 1996; 1: 45-73.

[2] Lade PV, Yamamuro JA, Bopp PA. Influence of time effects on instability of granular materials, Computers and Geotechnics 1997; 20(3-4):179-193.

[3] Tatsuoka F, Jardine RJ, Lo Presti D, Di Benedetto H, Kodaka, T. Characterising of the Pre-Failure Deformation Properties of Geomaterials. Proceedings of the $14^{\text {th }}$ International Conference on Soil Mechanics and Foundation Engineering, Hamburg, Germany, 2129-2164, 1997.

[4] Di Benedetto H, Tatsuoka F. Small strain behavior of geomaterials: modelling of strain rate effects. Soils and Foundations 1997; 37(2): 127-138.

[5] Di Benedetto H, Ibraim E, Cazacliu B. Time dependent behaviour of sand. Proceedings of the $2^{\text {nd }}$ International Symposium on Pre-failure Deformation Characteristics of Geomaterials, Torino, Italy, Jamiolkowski M et al. (eds), 459-466, 1999.

[6] Di Benedetto H, Tatsuoka F, Lo Presti D, Sauzéat C, Geoffroy H. Time effects on the behaviour of geomaterials, Deformation Characteristics of Geomaterials: Recent Investigations and Prospects, Di Benedetto et al. (eds). Taylor \& Francis Group, London, 59-123, 2005.

[7] Augustesen A, Liingaard M, Lade PV. Evaluation of Time-Dependent Behavior of Soils. International Journal of Geomechanics 2004; 4(3):137-156.

[8] Pham Van Bang D, Di Benedetto H, Duttine A, Ezaoui A. Viscous behaviour of dry sand. International Journal for Numerical and Analytical Methods in Geomechanics 2007; 31: 16311658.

[9] Perzyna P. The constitutive equations for rate sensitive plastic materials. Quarterly of Aapplied Mathematics 1963; 20(4): 321-332.

[10] Duvaut G, Lion JL. Les inequations en mechanique et en physique. Dunod:Paris,1972.

[11] Wang WM, Sluys LJ, De Borst R. Viscoplasticity for instabilities due to strain softening and strain-rate softening. International Journal for Numerical Methods in Engineering 1997; 40 (20): 3839-3864.

[12] Runesson K, Ristinmaa M, Mähler L. A comparison of viscoplasticity formats and algorithms. Mech. Cohesive frictional Mater 1999; 4(1): 75-98.

[13] Carosio A, Willam K, Etse G. On the consistency of viscoplastic formulations. International Journal of Solids and Structures 2000; 37(48-50): 7349-7369. 
[14] Heeres O, Suiker A, de Borst R. A comparison between the Perzyna viscoplastic model and the Consistency viscoplastic model. European Journal of Mechanics. A/Solids 2002; 21: 1-12.

[15] Di Benedetto H, Tatsuoka F, Ishihara M. Time-dependent shear deformation characteristics of sand and their constitutive modelling. Soils and Foundations 2002; 42(2): 1-22.

[16] Tatsuoka F, Uchimura T, Hayano K, Koseki J, Di Benedetto H, Siddiquee MS. A. Timedependent deformation characteristics of stiff geomaterials in engineering practice. In $\mathrm{M}$. Jamiolkowski, R. Lancellotta, \& D. Lo Presti (Eds.), Pre-Failure Deformation Characteristics of Geomaterials, 1161-1250, 1999.

[17] Perzyna P. Fundamental problems in viscoplasticity. Advances in Applied Mechanics 1966; 9: 243-377.

[18] Borja RI. Modeling the monotonic and cyclic viscoplastic soil behavior. Proceedings of the second International Conference on recent advances in geotechnical earthquake engineering and soil dynamics (ed. S. Prakash), vo1 1.14: 37-40. Rolla, MO, USA: Missouri University of Science and Technology, 1991.

[19] di Prisco C, Imposimato S, Vardoulakis I. Mechanical modelling of drained creep triaxial tests on loose sand. Géotechnique 2000; 50(1): 73-82.

[20] di Prisco C, Imposimato, S. Static liquefaction of a saturated loose sand stratum, International Journal of Solids and Structures 2002; 39: 3523-3541.

[21] Yin ZY, Chang CS, Karstunen M, Hicher PY. An anisotropic elastic-viscoplastic model for soft clays. International Journal of Solids and Structures 2010; 5: 665-677.

[22] Oka F, Adachi T, Yashima A. Instability of an elastoviscoplastic constitutive model for clay and strain localization. Mechanics of Materials 1994; 18: 119-129.

[23] Pisanò F, di Prisco C. A stability criterion for elasto-viscoplastic constitutive relationships. International Journal for Numerical and Analytical Methods in Geomechanics 2015; 40(1): 141156.

[24] Marinelli F, Buscarnera G. Instability criteria for quasi-saturated viscous soils. International Journal for Numerical and Analytical Methods in Geomechanics 2017; DOI: 10.1002/nag.2746.

[25] Marinelli F, Pisanò F, di Prisco C, Buscarnera G. Model-based interpretation of undrained creep instability in loose sands. Géotechnique 2017; 1-14.

[26] Needleman A. Material rate dependence and mesh sensitivity on localization problems. Computer Methods in Applied Mechanics and Engineering 1988; 67: 69-86.

[27] Loret B, Prevost JH. Dynamic strain localization in fluid-saturated porous media. Journal of Engineering Mechanics 1991; 117(4):907-922.

[28] Sluys LJ. Wave propagation, localisation and dispersion in softening solids. Ph.D. Thesis, Delft University of Technology, 1992.

[29] Schrefler B A, Zhang HW, Sanavia L. Fluid-structure interaction in the localisation of Saturated porous media, Zeitschrift für Angewandte Mathematik und Mechanik (Journal of Applied Mathematics and Mechanics, Z. Angew. Math. Mech.) 1999; 79(7): 481-484.

[30] Ehlers W, Graf T, Ammann M. Deformation and localization analysis of partially saturated soil. 
[31] Rice JR. The localisation of plastic deformation. Proceedings of the 14th IUTAM Congress, Koiter W.T. (ed.), Delft (North Holland, Amsterdam), 207-220, 1976.

[32] di Prisco C, Imposimato S. Nonlocal numerical analyses of strain localization in dense sand. Mathematical and Computer Modelling 2003; 37: 497-506.

[33] Lazari M, Sanavia L, Schrefler BA. Local and non-local elasto-viscoplasticity in strain localization analysis of multiphase geomaterials. International Journal for Numerical and Analytical Methods in Geomechanics 2015; 39:1570-1592.

[34] Buscarnera G, Nova R. An elastoplastic strain hardening model for soil allowing for hydraulic bonding-debonding effects. International Journal for Numerical and Analytical Methods in Geomechanics 2009; 33(8): 1055-1086.

[35] Jommi C. Remarks on the constitutive modelling of unsaturated soils. Experimental Evidence and Theoretical Approaches in Unsaturated Soils, In Proceedings of an International Workshop, Trento, 139-153, 2000.

[36] Jommi C, di Prisco C. A simple theoretical approach for modelling the mechanical behaviour of unsaturated soils (in Italian). In Proceedings of the Conference "Il ruolo dei fluidi nei problemi di Ingegneria geotecnica", 1: 167-188, 1994.

[37] Nova R, Castellanza R, Tamagnini C. A constitutive model for bonded geomaterials subject to mechanical and/or chemical degradation. International Journal for Numerical and Analytical Methods in Geomechanics 2003; 27: 705-732.

[38] di Prisco C, Imposimato S, Aifantis EC. A visco-plastic constitutive model for granular soils modified according to non-local and gradient approaches. International Journal for Numerical and Analytical Methods in Geomechanics 2002; 26: 121-138.

[39] Schrefler BA. The finite element method in soil consolidation (with applications to surface subsidence), PhD. Thesis, University College of Swansea, 1984.

[40] Borja RI, Tamagnini C, Amorosi A. Coupling plasticity and energy-conserving elasticity models for clay. Journal of Geotechnical and Geoenvironmental Engineering 1997; 123(10): 948-957.

[41] Tamagnini C, Castellanza R, Nova R. A generalized backward Euler algorithm for the numerical integration of an isotropic hardening elastoplastic model for mechanical and chemical degradation of bonded geomaterials. International Journal for Numerical Methods in Engineering 2002; 26: 963-1001.

[42] Lagioia R, Puzrin A, Potts D. A new versatile expression for yield e plastic potential surfaces. Computers and Geothecnics 1996; 19(3): 171-191.

[43] Gudehus G. Elastoplastische stoffgleichungen für trochkenen sand. Infenieyr-Archiv 1973; 42: $151-169$.

[44] Pisanò F. Seismic Performance of Infinite Earth Slopes: Numerical Modelling, Constitutive Issues and Theoretical Considerations, Ph.D. Thesis, Politecnico di Milano, Italy, 2011.

[45] di Prisco C, Pastor M, Pisanò F. Shear wave propagation along infinite slopes: A theoretically based numerical study. International Journal for Numerical and Analytical Methods in Geomechanics 2012; 36(5): 619-642. 
[46] Uriel S. Intrinsic dynamic of the quasi-static mechanics of granular soils. In Numerical Methods in Soil and Rock Mechanics, Borm G, Meissher H (eds). Institut fu Bondenmechanik und Felsmechanik der Universitat Karlsruhe: Karlsruhe: 61-70, 1975.

[47] Been K, Jefferies MG. A state parameter for sands. Géotechnique 1985; 35(2): 99-112.

[48] Wood DM, Belkheir K. Strain softening and state parameter for sand modelling. Géotechnique 1994; 44(2): 335-339.

[49] Manzari M, Dafalias Y. A critical state two-surface plasticity model for sands. Géotechnique 1997; 47(2): 255-272.

[50] Gajo A, Wood DM. Seven-Trent sand: a kinematic-hardening constitutive model: the q-p formulation. Géotechnique 1999; 49 (5): 595-614.

[51] Manzanal D, Fernández-Merodo JA, Pastor M. Generalized plasticity state parameter-based modelling of saturated and unsaturated soils. Part I: saturated state. International Journal for Numerical and Analytical Methods in Geomechanics 2011; 35: 1347-1362.

[52] Lazari M. Finite element regularization for post localized bifurcation in variably saturated media, Ph.D. Thesis, University of Padova, Italy, 2016.

[53] Jirasék M. Objective modeling of strain localization. Revue française de génie civil 2002; 6: 119 1132.

[54] Jirásek M, Rolshoven S. Comparison of integral-type nonlocal plasticity models for strainsoftening materials. International Journal of Engineering Science 2003; 41: 1553-1602.

[55] Lu X, Bardet JP, Huang M. Spectral analysis of nonlocal regularization in two-dimensional finite element models. International Journal for Numerical and Analytical Methods in Geomechanics 2012; 36: 219-235.

[56] di Prisco C, Stupazzini M, Zambelli C. Nonlinear SEM numerical analyses of dry dense sand specimens under rapid and dynamic loading. International Journal for Numerical and Analytical Methods in Geomechanics 2007; 31(6): 757-788.

[57] Murianni A, Di Prisco C, Federico A. Numerical stability of non-local viscoplastic FEM analyses for the study of localisation processes. Geomechanics and Geoengineering 2013; 8(4): 215-228.

[58] Lazari M, Sanavia L, Schrefler BA. Finite element modelling of shear bands in porous media by means of non-local viscoplasticity. COMPLAS XIII: proceedings of the XIII International Conference on Computational Plasticity: fundamentals and applications, Barcelona: CIMNE, $2015,140-150$.

[59] Lewis RW, Schrefler BA. The Finite Element Method in the Static and Dynamic Deformation and Consolidation of Porous Media. John Wiley \& Sons, Chichester, 1998.

[60] Gawin D, Schrefler BA. Thermo-hydro-mechanical analysis of partially saturated porous materials. Engineering Computations 1996; 13(7): 113-143.

[61] Sanavia L, Pesavento F, Schrefler BA. Finite element analysis of non-isothermal multiphase geomaterials with application to strain localization simulation. Computational Mechanics 2006; 37: 331-348.

[62] Sanavia L. Numerical Modelling of a Slope Stability Test by Means of Porous Media Mechanics. 
[63] Gawin D, Sanavia L. A unified approach to numerical modelling of fully and partially saturated porous materials by considering air dissolved in water. Computer Modeling in Engineering and Sciences 2009; 53: 255-302.

[64] Gawin D, Sanavia L. Simulation of cavitation in water saturated porous media considering effects of dissolved air. Transport in Porous Media 2010; 81: 141-160.

[65] Kakogiannou E, Sanavia L, Nicot F, Darve F, Schrefler BA. A porous media finite element approach for soil instability including the second-order work criterion, Acta Geotechnica 2016; 11(4): 805-825.

[66] Cao TD, Sanavia L, Schrefler BA. A thermo-hydro-mechanical model for multiphase geomaterials in dynamics with application to strain localization simulation. International Journal for Numerical Methods in Engineering 2016; 107(4): 312-337.

[67] Sheng D, Sloan SW, Gens A, Smith DW. Finite element formulation and algorithms for unsaturated soils. Part I: Theory. International Journal for Numerical and Analytical Methods in Geomechanics 2003; 27(9): 745-765.

[68] Cattaneo F, Della Vecchia G, Jommi C. Evaluation of numerical stress-point algorithms on elastic-plastic models for unsaturated soils with hardening dependent on the degree of saturation. Computers and Geotechnics 2014; 55: 404-415.

[69] Daouadji A, Al Gali H, Darve F, Zeghloul A. Instability in Granular Materials: Experimental Evidence of Diffuse Mode of Failure for Loose Sands. Journal of Engineering Mechanics 2010; 136(5): 575-588.

[70] Mokni M, Desrues J. Strain localisation measurements in undrained plane-strain biaxial tests on hostun RF sand. Mechanics of Cohesive-Frictional Materials 1998; 4: 419-441.

[71] Alesani M, Fantini S. Stability analysis of the mechanical behaviour of granular materials under undrained conditions (in Italian). Master Thesis, Politecnico di Milano (Italy), 1998.

[72] Desrues J, Viggiani G. Strain localization in sand: an overview of the experimental results obtained in Grenoble using stereophotogrammetry. International Journal for Numerical and Analytical Methods in Geomechanics 2004; 28: 279-321.

[73] Panteghini A, Lagioia R. An extended modified cam-clay yield surface for arbitrary meridional and deviatoric shapes retaining full con-vexity and double homothety. Géotechnique 2018; 68(7): $590-601$.

[74] Panteghini A, Lagioia R. An approach for providing quasi-convexity to yield functions and a generalized implicit integration scheme for isotropic constitutive models based on 2 unknowns. International Journal for Numerical and Analytical Methods in Geomechanics 2018; 42(6): 829855.

[75] di Prisco C, Imposimato S, Nova R. Sand specimen undrained mechanical response to instantaneous load increments. Proceedings of Plasticity'99, $7^{\text {th }}$ International Symposium on plasticity and its current applications, Cancun, Mexico, 557-560, 1999. 
Table 1: List of constitutive parameters.

\begin{tabular}{|ll|}
\hline \multicolumn{1}{|c|}{ Hyperelastic law } \\
\hline$\hat{k}$ & slope of the swelling isotropic compression line \\
a & deviatoric-volumetric elastic coupling parameter \\
$G_{0}$ & representative elastic shear modulus \\
$p_{r}$ & reference pressure \\
\hline \multicolumn{2}{c|}{$\quad$ Yield function } \\
\hline$m_{f}$ & yield function shape parameter \\
$\alpha_{f}$ & yield function shape parameter \\
$M_{c f}$ & stress obliquity for local yield locus maximum in triaxial compression \\
$M_{e f}$ & stress obliquity for local yield locus minimum in triaxial extension \\
\hline \multicolumn{2}{c|}{$\quad$ Plastic potential } \\
\hline$m_{g}$ & plastic potential shape parameter \\
$\alpha_{g}$ & plastic potential shape parameter \\
$M_{c g}$ & stress obliquity for isochoric plastic flow in triaxial compression \\
$M_{e g}$ & stress obliquity for isochoric plastic flow in triaxial extension \\
\hline \multicolumn{2}{c}{$\quad$ Hardening rule } \\
\hline$\rho_{s}$ & mechanical hardening of pre-consolidation pressure \\
$\xi_{s}$ & dilatancy at failure \\
$r_{s w}$ & hydraulic hardening of pre-consolidation pressure \\
\hline \multicolumn{2}{c}{$\quad$ Viscous nucleus } \\
\hline$\gamma$ & fluidity parameter \\
$\alpha$ & viscous nucleus shape parameter \\
\hline
\end{tabular}

Table 2: Literature experimental tests on Hostun sand used for model calibration/validation.

\begin{tabular}{|c|c|c|c|c|}
\hline Test name & Drainage & $p_{0}^{\prime}[\mathrm{kPa}]$ & $\boldsymbol{e}_{0}[-]$ & Reference \\
\hline \multicolumn{5}{|c|}{ Triaxial compression tests } \\
\hline hos 011 & Drained & 300 & 0.574 & \multirow{8}{*}{ Gajo and Wood (1999) [50] } \\
\hline hos027 & Drained & 200 & 0.578 & \\
\hline hosfl10 & Drained & 300 & 0.800 & \\
\hline hosfl11 & Drained & 300 & 0.897 & \\
\hline $\mathrm{d} 4$ & Drained & 300 & 0.945 & \\
\hline hosfl14 & Drained & 50 & 0.838 & \\
\hline hflw10 & Drained & 600 & 0.822 & \\
\hline batr02 & Undrained & 200 & 0.940 & \\
\hline
\end{tabular}




\begin{tabular}{|c|c|c|c|c|}
\hline batr06 & Undrained & 200 & 0.830 & \\
\hline alert9 & Undrained & 200 & 0.666 & \\
\hline 400.95 & Drained & 400 & 0.950 & Pham Van Bang et al. (2007) [8] \\
\hline $\mathrm{CD}-1$ & Drained & 100 & 1.052 & \\
\hline $\mathrm{CD}-2$ & Drained & 300 & 0.954 & \\
\hline $\mathrm{CD}-3$ & Drained & 750 & 1.010 & Daouadji et al. (2010) [69] \\
\hline ICU-1 & Undrained & 100 & 1.086 & \\
\hline ICU-2 & Undrained & 300 & 1.060 & \\
\hline ICU-3 & Undrained & 750 & 1.083 & \\
\hline \multicolumn{5}{|c|}{ Biaxial compression test } \\
\hline SHFND05 & Undrained & 800 & 0.945 & Mokni and Desrues (1998) [70] \\
\hline \multicolumn{5}{|c|}{ Creep tests } \\
\hline T100a & Drained & 100 & 0.950 & di Prisco and Imposimato (1996) [1] \\
\hline $80.71 \_\mathrm{ci}$ & Drained & 80 & 0.710 & Pham Van Bang et al. (2007) [8] \\
\hline $20 \mathrm{DP} 13$ & Undrained & 100 & 0.900 & Alesani and Fantini (1998) [71] \\
\hline
\end{tabular}

Table 3: $D_{r}$-insensitive constitutive parameters.

\begin{tabular}{lll}
\hline Elastic law & Yield locus & Plastic potential \\
\hline $\mathrm{a}=0.0$ & $\alpha_{f}=0.99$ & $\alpha_{g}=0.24$ \\
$k=0.0046$ & $m_{f}=1.10$ & $m_{g}=1.10$ \\
$p_{r}=1 \mathrm{kPa}$ & $M_{c f}=0.52$ & $M_{c g}=1.28$ \\
& $M_{e f}=0.44$ & $M_{e g}=1.0$ \\
\hline
\end{tabular}

600

601 Table 4: $D_{r}$-dependent constitutive parameters.

\begin{tabular}{lllll}
\hline Sample Density & $G_{0}[\mathrm{kPa}]$ & $\rho_{s}[-]$ & $\xi_{s}[-]$ & $r_{s w}[-]$ \\
\hline Loose & 14000 & 111 & 0.00 & 5.45 \\
Dense & 45000 & 2000 & 0.480 & 0.32 \\
Limit void ratios & $\mathrm{e}_{\max }$ & 1.041 & $\mathrm{e}_{\min }$ & 0.58 \\
\hline
\end{tabular}

602 
Table 5: Viscous parameters for loose Hostun sand (from drained creep tests).

\begin{tabular}{lll}
\hline Viscous nucleus & $\gamma\left[\mathrm{s}^{-1}\right]$ & $\alpha[-]$ \\
\hline linear & $4 \cdot 10^{-9}$ & 1.0 \\
exponential & $2 \cdot 10^{-14}$ & 28.90 \\
\hline
\end{tabular}

604

605

606

Table 6: Viscous parameters for dense Hostun sand (from drained creep tests).

\begin{tabular}{lll}
\hline Viscous nucleus & $\gamma\left[\mathrm{s}^{-1}\right]$ & $\alpha[-]$ \\
\hline linear & $1 \cdot 10^{-8}$ & 1.0 \\
exponential & $2 \cdot 10^{-10}$ & 28.90 \\
\hline
\end{tabular}

607

608

609

Table 7: Viscous parameters for undrained conditions (exponential viscous nucleus).

\begin{tabular}{lll}
\hline Sample density & $\gamma\left[\mathrm{s}^{-1}\right]$ & $\alpha[-]$ \\
\hline TXU - Loose & $2 \cdot 10^{-5}$ & 28.90 \\
TXU - Dense & $2 \cdot 10^{-4}$ & 28.90 \\
\hline
\end{tabular}

610 